\title{
Perturbations of Respiratory Rhythm and Pattern by Disrupting Synaptic Inhibition within Pre-Bötzinger and Bötzinger Complexes ${ }^{1,2,3}$
}

(D) Vitaliy Marchenko, ${ }^{1, *}$ Hidehiko Koizumi, ${ }^{2, *}$ Bryan Mosher, ${ }^{2}$ №ohiro Koshiya, ${ }^{2}$ Mohammad F. Tariq, ${ }^{2}$ (1)Tatiana G. Bezdudnaya, ${ }^{1}$ Ruli Zhang, ${ }^{2}$ Yaroslav I. Molkov, ${ }^{3}$ (1) llya A. Rybak, ${ }^{1}$ and Jeffrey C. Smith ${ }^{2}$

DOI:http://dx.doi.org/10.1523/ENEURO.0011-16.2016

${ }^{1}$ Department of Neurobiology and Anatomy, Drexel University College of Medicine, Philadelphia, Pennsylvania 19129, ${ }^{2}$ Cellular and Systems Neurobiology Section, National Institute of Neurological Disorders and Stroke, National Institutes of Health, Bethesda, Maryland 20892, and ${ }^{3}$ Department of Mathematics and Statistics, Georgia State University, Atlanta, Georgia 30302

\section{Visual Abstract}

\section{Gabazine + Strychnine in BötC (in vivo)}

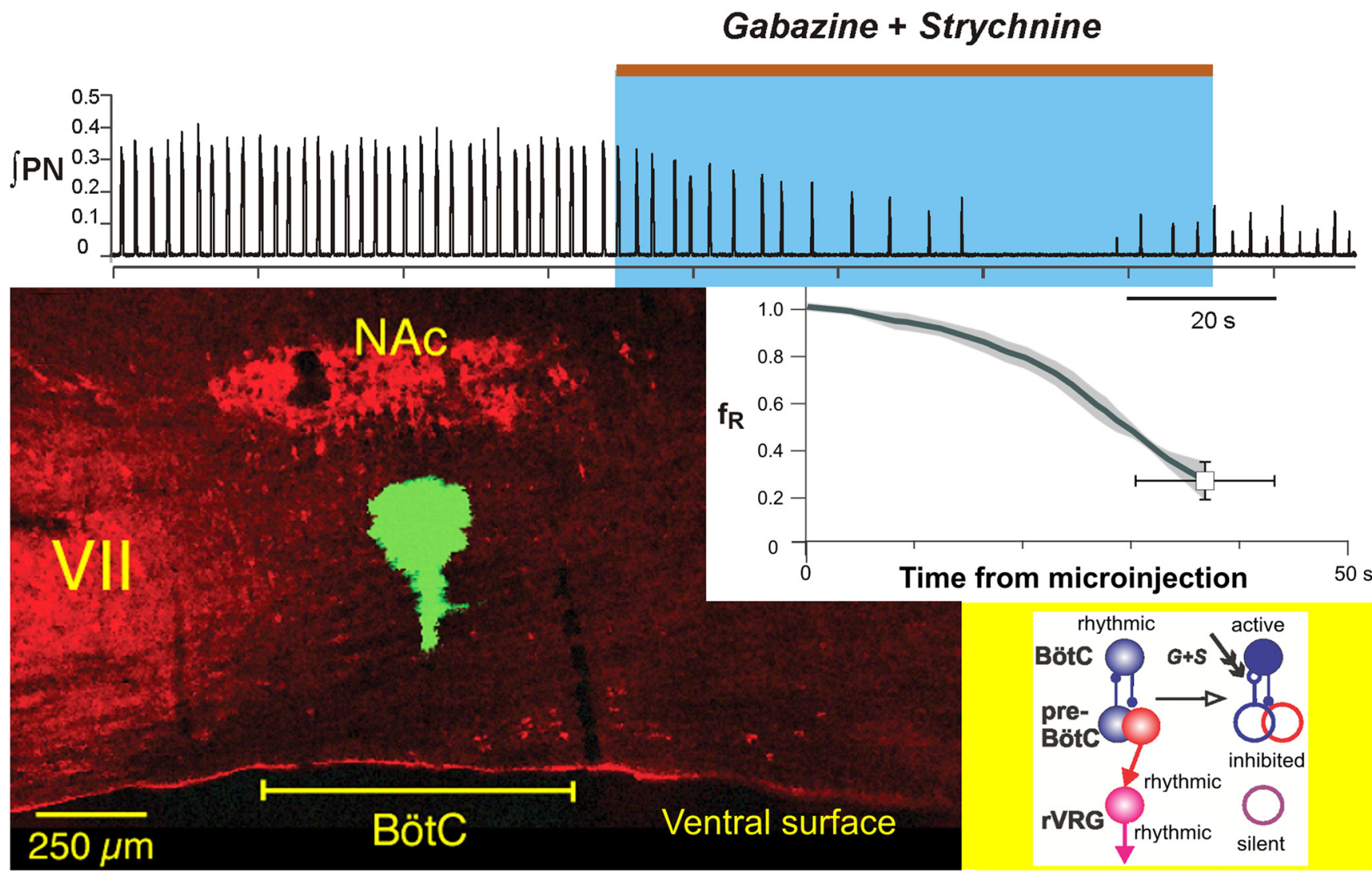

The pre-Bötzinger (pre-BötC) and Bötzinger (BötC) complexes are the brainstem compartments containing interneurons considered to be critically involved in generating respiratory rhythm and motor pattern in mammals. Current models postulate that both generation of the rhythm and coordination of the inspiratory-expiratory pattern involve inhibitory synaptic interactions within and between these regions. Both regions contain glycinergic and 
GABAergic neurons, and rhythmically active neurons in these regions receive appropriately coordinated phasic inhibition necessary for generation of the normal three-phase respiratory pattern. However, recent experiments attempting to disrupt glycinergic and GABAergic postsynaptic inhibition in the pre-BötC and BötC in adult rats in vivo have questioned the critical role of synaptic inhibition in these regions, as well as the importance of the BötC, which contradicts previous physiological and pharmacological studies. To further evaluate the roles of synaptic inhibition and the BötC, we bilaterally microinjected the $\mathrm{GABA}_{A}$ receptor antagonist gabazine and glycinergic receptor antagonist strychnine into the pre-BötC or BötC in anesthetized adult rats in vivo and in perfused in situ brainstem-spinal cord preparations from juvenile rats. Muscimol was microinjected to suppress neuronal activity in the pre-BötC or BötC. In both preparations, disrupting inhibition within pre-BötC or BötC caused major site-specific perturbations of the rhythm and disrupted the three-phase motor pattern, in some experiments terminating rhythmic motor output. Suppressing BötC activity also potently disturbed the rhythm and motor pattern. We conclude that inhibitory circuit interactions within and between the pre-BötC and BötC critically regulate rhythmogenesis and are required for normal respiratory motor pattern generation.

Key words: Bötzinger complex; brainstem; central pattern generation; pre-Bötzinger complex; respiration; synaptic inhibition

\section{Significance Statement}

Defining functional roles of postsynaptic inhibition in respiratory and other mammalian central pattern generation circuits is a longstanding problem. Inhibitory circuit interactions within and between the brainstem respiratory pre-BötC and BötC have been proposed to be critically involved in normal rhythm and motor pattern generation. A fundamental role of postsynaptic inhibition in these regions has been questioned in recent experiments attempting to pharmacologically disrupt this inhibition. To resolve this contradiction, we applied similar approaches of microinjecting selective pharmacological antagonists of $\mathrm{GABA}_{A}$ ergic and glycinergic receptor-mediated inhibition in the pre-BötC and BötC in rats. Our results demonstrate large, site-specific perturbations of respiratory rhythm and motor pattern including disruption of rhythmic motor output and thus confirm the critical role of inhibitory circuit interactions.

\section{Introduction}

Rhythmic movements such as breathing and locomotion are produced by central pattern generator (CPG) networks containing interacting excitatory and inhibitory circuits. These circuits are the neural substrates for producing motor behavior (Grillner, 2006) and defining their specific roles in rhythmic motor pattern generation is key to understanding the functional operation of CPGs. Here we have addressed the longstanding problem of defining roles of inhibitory circuits in core structures of the mammalian brainstem respiratory CPG.

Received January 16, 2016; accepted April 18, 2016; First published May 02, 2016.

${ }^{1}$ The authors report no conflict of interest.

${ }^{2}$ Author contributions: V.M., H.K., I.A.R., and J.C.S. designed research; V.M., H.K., B.M., T.G.B., and R.Z. performed research; H.K., N.K., M.F.T., R.Z., and Y.I.M. analyzed data; I.A.R. and J.C.S. wrote the paper.

${ }^{3}$ This work was supported in part by the Intramural Research Program of the National Institutes of Health (NIH), National Institute of Neurological Disorders and Stroke, and NIH Grants R01 NS069220 and R01 AT008632

*V.M. and H.K. contributed equally to this work.

Correspondence should be addressed to Dr. Jeffrey C. Smith, 49 Convent Drive, Room 2A10, NINDS, NIH, Bethesda, MD 20892. E-mail: smithj2@helix. nih.gov.

DOI:http://dx.doi.org/10.1523/ENEURO.0011-16.2016

Copyright (C) 2016 Marchenko et al.

This is an open-access article distributed under the terms of the Creative Commons Attribution 4.0 International, which permits unrestricted use, distribution and reproduction in any medium provided that the original work is properly attributed.
The respiratory neural pattern under normal conditions includes three phases: inspiration, post-inspiration, and late expiration (Richter, 1996; Richter and Smith, 2014). The kernel of the respiratory CPG located in the ventral respiratory column of the medulla includes two key compartments, the pre-Bötzinger (pre-BötC) and Bötzinger (BötC) complexes (Alheid and McCrimmon, 2008; Smith et al., 2009, 2013). The pre-BötC contains a heterogeneous population of excitatory neurons, including cells with intrinsic bursting properties, and excitatory synaptic interconnections that generate rhythmic inspiratory activity and drive inspiratory motor output. This excitatory population, when isolated in slices in vitro, generates inspiratory bursting activity (Smith et al., 1991; Koshiya and Smith, 1999) that persists after disrupting synaptic inhibition (Johnson et al., 2001). In addition, the pre-BötC contains GABAergic and glycinergic neuron populations (Kuwana et al., 2006; Winter et al., 2009; Morgado-Valle et al., 2010; Koizumi et al., 2013) providing phasic inspiratory inhibition widely distributed in the brainstem including inhibition of BötC expiratory neurons. In turn, BötC postinspiratory and expiratory neurons provide phasic expiratory inhibition (Jiang and Lipski, 1990; Tian et al., 1999a,b; Ezure et al., 2003a,b) including to pre-BötC inspiratory neurons to coordinate generation of expiratory and inspiratory phases.

The specific contributions of the intrinsic excitatory bursting mechanisms in the pre-BötC, and inhibitory net- 
work interactions between pre-BötC and BötC, to respiratory rhythm and motor pattern generation are not clearly understood and this issue is continuously debated. Early theoretical models, based entirely on network inhibitory interactions, could not explain the maintenance of rhythm after blockade of synaptic inhibition in vitro. Alternatively, the pure autorhythmic excitatory network models, developed to explain the in vitro data, could not account for many behaviors observed in vivo, such as the HeringBreuer inspiratory inhibitory and other respiratory reflexes and the coordinated generation of multiple respiratory phases. Also, these models could not reproduce apneusis, a breathing pattern characterized by a significantly prolonged inspiration alternating with short expiratory intervals (Lindsey et al., 2012).

To resolve this problem, more complicated models have been developed (Rybak et al., 2004, 2007; Smith et al., 2007, 2009, 2013) hypothesizing that: (1) the preBötC, although capable of autonomous generation of rhythmic bursting when isolated in vitro, is embedded in the larger respiratory network where its activity is controlled by interactions with other brainstem compartments, including inputs from excitatory RTN/pFRG neurons, and from the inhibitory neuron populations in BötC, and (2) both the intrinsic bursting of pre-BötC inspiratory neurons and inhibitory interactions between the neural populations in pre-BötC and BötC are fundamentally involved in generating the normal rhythmic respiratory pattern. Disruption of inhibition in these circuits would lead either to switching to the intrinsic rhythmic activity originating within the pre-BötC, or to sustained or apneustic-like activity.

This concept was challenged by a recent study in the anesthetized rat using targeted pharmacological blockade of fast inhibitory neurotransmission (Janczewski et al., 2013) from which it was concluded that: (1) the BötC does not play a role in respiratory rhythm/pattern generation, and (2) inhibition within the pre-BötC and BötC is not required for generating a normal breathing rhythm and pattern.

The present study was focused on resolving this contradiction and further evaluating roles of inhibitory interactions in pre-BötC and BötC in rhythm generation and shaping respiratory pattern. Our experiments were performed using two distinct preparations, the anesthetized, vagotomized adult rat, and the arterially perfused in situ brainstem-spinal cord preparation of juvenile rat. Specific pharmacological blockers of glycinergic (strychnine) and $\mathrm{GABA}_{A}$ ergic (gabazine) receptor-mediated inhibition were selectively microinjected into the pre-BötC or BötC and perturbations of the respiratory frequency and phases were evaluated. In addition, microinjections of the GABA receptor agonist muscimol were used to inhibit neural activity in each compartment. Our results were not consistent with those reported by Janczewski et al. (2013). Disrupting inhibition within pre-BötC or BötC, as well as inhibiting BötC activity, caused major perturbations of the respiratory frequency and three-phase pattern. Moreover, blocking inhibition within the BötC lead to apnea confirming the critical role of inhibitory interactions between pre-
BötC and BötC. Our results are consistent with previous proposals of the important role of inhibitory interactions within and between pre-BötC and BötC in generating and shaping the respiratory pattern.

\section{Materials and Methods}

\section{Animal procedures}

All experimental procedures used in this study were approved by either the NINDS Animal Care and Use Committee, or the Drexel University Institutional Animal Care and Use Committee, which oversees Drexel University's AAALAC International-accredited animal program. All electrophysiological recording and pharmacological microinjection experiments were performed via a surgically exposed ventral brainstem for access to the ventrolateral medullary pre-BötC and BötC regions (Fig. 1).

\section{Surgical procedures in adult rats in vivo}

Spontaneously breathing, adult male Sprague-Dawley rats $(340-380 \mathrm{~g})$ were anesthetized with isoflurane vaporized in $\mathrm{O}_{2}$ (Matrix; 4-5\% induction, 1.75-2.0\% maintenance) via a snout mask. Anesthetic depth was maintained at a level at which withdrawal reflexes, as well as changes in heart rate and blood pressure in response to pinching the distal hind limbs, were absent. After tracheotomy with a glass tube, animals were artificially ventilated with the same gas mixture $\left(60 \mathrm{~min}^{-1}, 2.5-3.0 \mathrm{ml}\right.$ tidal volume; Columbus Apparatus rodent ventilator). Electrocardiogram was measured via three small subcutaneous electrodes using conventional amplification and filtering (Neurolog; Digitimer) and monitored using an audio amplifier (model AM10; Grass Instruments) and oscilloscope (Tektronix). One femoral artery and vein were cannulated for measurement of arterial pressure and infusion of drugs/saline, respectively. During all surgical procedures, rectal temperature was maintained at $37.0 \pm$ $0.1^{\circ} \mathrm{C}$ via a servo-controlled heating blanket coupled to a rectal thermometer (Harvard Apparatus). The phrenic nerve $(P N)$ was prepared for recording by dissecting the nerves free from the surrounding tissue. Ventral neck muscles (cleidomastoideus, sternomastoideus, sternohyoideus, omohypides, and digastricus), infrathyroid portions of the trachea and esophagus were removed. The body of the 1st neck vertebra (atlas) and base portion of occipital bone were removed to expose the ventral medulla and the axo-occipital membrane was cut. The dura was then opened using iridectomy scissors and residual bleeding from lateral epidural sinus was arrested by applying small pieces of gelfoam (USP, Pharmacia) soaked with thrombin solution $\left(50 \mathrm{U} \cdot \mathrm{ml}^{-1} \mathrm{USP}\right.$, Biopharm Laboratories) dissolved in artificial CSF (aCSF).

All animals were vagotomized and baro- and chemoreceptor denervated via bilateral transection of the carotid sinus nerves to prevent cardiorespiratory reflex influences on motor nerve outputs (Richter and Seller, 1975; Grundy et al., 1986; Hopp and Seagard, 1998; Virkki et al., 2007; Baekey et al., 2010). A bilateral pneumothorax was performed before electrophysiological recording to eliminate 

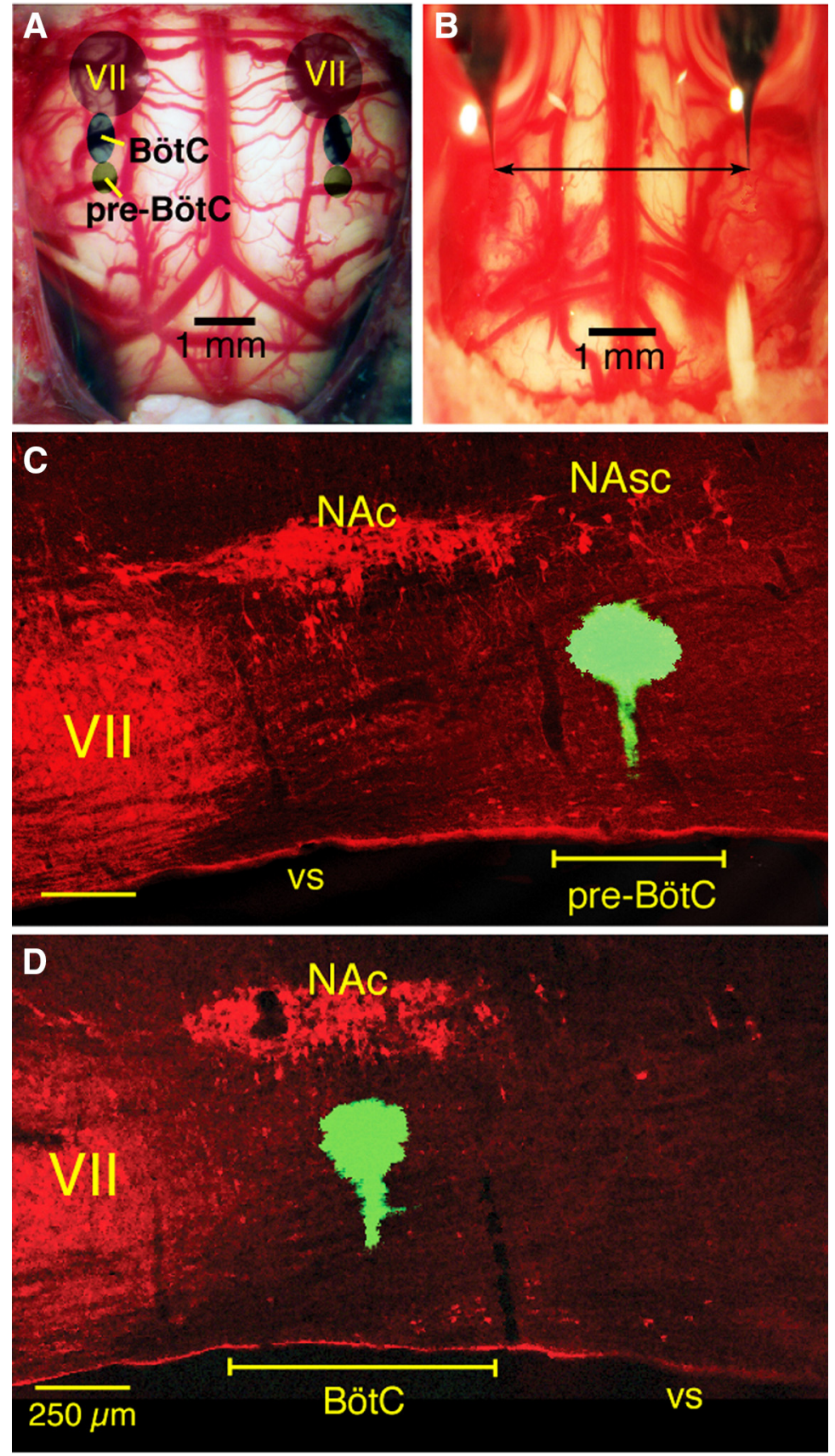

Figure 1. Ventral view of the adult rat medulla and histology illustrating targeted sites for pharmacology experiments in vivo. $\boldsymbol{A}$, Photograph of adult rat brainstem ventral surface as exposed in the in vivo experimental preparations with an overview of targeted locations (caudal to facial motor nucleus, VII) of BötC and pre-BötC as routinely identified by electrophysiological mapping of neuronal activity profiles in the present experiments. $\boldsymbol{B}$, Photomicrograph of ventral medullary surface and bilaterally arranged pipettes (blue dye-filled for visualization) as typically configured for near perpendicular penetrations of the ventral surface for simultaneous microinjections. $\boldsymbol{C}, \boldsymbol{D}$, Confocal microscopic images of parasagittal histologic sections (50 $\mu \mathrm{m}$ thick) showing, respectively, examples of targeted sites for microinjection of inhibitory antagonists in the pre-BötC ventral to the semicompact subdivision of nucleus ambiguous (NAsc), and in the BötC ventral to the compact subdivision of nucleus ambiguus (NAc). Targeted sites are marked by microinjected solution of fluorescent microspheres (green). NA and VII motoneurons are immunolabeled by ChAT antibody (red). Rostrocaudal spatial extent of the pre-BötC and BotC compartments are indicated. vs, ventral surface.

lung inflation-related movement artifacts and chest wall mechanoreceptor feedback. A positive end-expiratory pressure of $1.0 \mathrm{~cm} \mathrm{H} \mathrm{H}_{2} \mathrm{O}$ was maintained to prevent lung atelectasis during expiration. Animals were paralyzed by an intravenous bolus injection ( $2 \mathrm{mg} / \mathrm{kg}$ ), followed by continuous infusion $(3-4 \mathrm{mg} / \mathrm{kg} / \mathrm{h}$ ), of vecuronium bromide (Abbott Laboratories) dissolved in Ringer-Locke solution. End-tidal $\mathrm{CO}_{2}$ was maintained between $5.0 \%$ and 
5.5\% (Capstar, CWE) by adjusting frequency of ventilation. If necessary, animals were continuously infused with Ringer-Locke solution (1.0-1.25\% body weight or 10.0$12.5 \mathrm{ml} / \mathrm{kg} / \mathrm{h}$ ) to maintain a stable mean arterial pressure of $85-95 \mathrm{mmHg}$.

\section{In situ arterially perfused brainstem-spinal cord preparation}

Experiments were also performed with the in situ arterially perfused brainstem-spinal cord preparations from juvenile rats (3-5 weeks old) as previously described (Paton, 1996; Smith et al., 2007). These preparations were studied because they provide the opportunity to investigate roles of synaptic inhibition in an un-anesthetized preparation generating the three-phase respiratory pattern that could be clearly identified from simultaneous recordings of spinal and cranial nerves, including prominent postinspiratory (post-l) discharge recorded from the central vagus nerve $(\mathrm{cVN})$. We note that $\mathrm{cVN}$ recordings from the normocapnic, vagotomized, carotid body denervated, and isoflurane anesthetized adult rats in vivo in our experiments do not routinely exhibit post-I discharge although neuronal recording in the BötC always shows post-I activity (see Fig. 3B), which is an important feature of medullary respiratory circuit activity. Furthermore, a number of theoretical models (Rybak et al., 2007; Smith et al., 2007; Rubin et al., 2009; Shevtsova et al., 2011, 2014; Richter and Smith, 2014) postulating roles of pre-BötC and BötC inhibitory circuits in respiratory rhythm and pattern generation have been based in part on experimental results obtained from these in situ preparations. It is therefore critical to test their roles in this preparation, and to compare results from the in vivo anesthetized adult rat preparations using a similar strategy for targeted disruption of synaptic inhibition in the pre-BötC and BötC.

Preheparinized (1000 units, given intraperitoneally) juvenile rats (Sprague-Dawley, 45-90 g; male) were anaesthetized deeply with $5 \%$ isoflurane and the portion of the body caudal to the diaphragm was removed. The head and thorax were immersed in ice-chilled carbogenated aCSF solution (in mM: $1.25 \mathrm{MgSO}_{4}, 1.25 \mathrm{KH}_{2} \mathrm{PO}_{4}, 5.0 \mathrm{KCl}$, $25 \mathrm{NaHCO}_{3}, 125 \mathrm{NaCl}, 2.5 \mathrm{CaCl}_{2}, 10$ dextrose, 0.1785 polyethylene glycol) and the rat was decerebrated at a precollicular level. The descending aorta, PN, and cVN were surgically isolated. As with the in vivo preparations, ventral neck muscles, infrathyroid portions of the trachea, the esophagus, and then the 1st neck vertebra and base portion of the occipital bone were removed to expose the ventral medulla. The axo-occipital membrane was cut, and the dura was then cut open. The preparation was transferred to a recording chamber and secured in a stereotaxic head frame ventral side up. The descending aorta was cannulated with a double-lumen catheter for perfusion and recording of perfusion pressure with a pressure transducer (Micron Instruments). Vecuronium bromide was added to the perfusate to block neuromuscular transmission ( $4 \mu \mathrm{g} / \mathrm{ml}$; SUN Pharmaceutical Industries). The perfusate was gassed with $95 \% \mathrm{O}_{2} / 5 \% \mathrm{CO}_{2}$ and maintained at $31^{\circ} \mathrm{C}$. Vasopressin $(200-400$ pm as re- quired; APP Pharmaceuticals) was added to the perfusate to raise and maintain perfusion pressure between 70 and $80 \mathrm{mmHg}$ (Paton et al., 2006). Unless stated, all chemicals were from Sigma-Aldrich.

\section{Electrophysiological recording in vivo}

With the rat in the supine position, the central ends of the cut PN were placed on bipolar silver hook electrodes for recording (10-5000 Hz bandpass; Neurolog, Digitimer) and immersed in a mineral oil pool formed by skin flaps. To identify precise locations of the BötC and pre-BötC the activity of medullary expiratory and (pre)inspiratory neurons was recorded (200-3000 Hz bandpass; Neurolog) by a ventral approach and glass (WPI) microelectrodes (tip outer diameter of 2-3 $\mu \mathrm{m}, 5-10 \mathrm{M} \Omega$ ) filled with $0.5 \mathrm{M} \mathrm{NaCl}$ and $2 \%$ pontamine sky blue. The pre-BötC is readily identified by a characteristic pattern of pre-inspiratory/ inspiratory (pre-1/I) activity (see Fig. $3 A$ ) and the BötC has a characteristic profile of post-I and augmenting expiratory (aug-E) activities (see Fig. 3B). The microelectrode was held in a three-dimensional stepper motor assembly (DC-3K, Märzhäuser), attached to the rail of the stereotaxic frame, and advanced in steps of 2.5-5.0 $\mu \mathrm{m}$. After mapping neuronal activity, the medullary surface was marked bilaterally by iontophoresis for $15 \mathrm{~min}$ of the pontamine sky blue dye $(-25 \mathrm{nA}, 0.125 \mathrm{~Hz}, 4 \mathrm{~s}$; Axoclamp $2 A)$ as a guide spot for insertion of drug microinjection pipettes. All electrophysiological signals were recorded simultaneously with expiratory $\mathrm{CO}_{2}$ level, arterial blood pressure, and lung inflation pressure, on the hard disk of a personal computer via a 16-bit analog-to-digital converter (PowerLab, AD Instruments, $10 \mathrm{kHz}$ sampling rate) and displayed continuously with software (Chart, AD Instruments).

\section{Electrophysiological recording in situ}

To monitor respiratory network activity and motor output in the in situ perfused brainstem-spinal cord preparations, we recorded with fire-polished glass suction electrodes inspiratory activity from $\mathrm{PN}$, and cVN inspiratory and post-inspiratory activity. Signals were amplified (50,000100,000×; CyberAmp 380, Molecular Devices), bandpass filtered $(0.3-2 \mathrm{kHz})$, digitized $(10 \mathrm{kHz}$ sampling rate) with an $A D$ converter [Cambridge Electronics Design (CED)], and then rectified and integrated digitally with Spike 2 software (CED). Extracellular population activity from pre-BötC or BötC respiratory neurons in the perfused in situ preparations was also recorded with a fine glass electrode (3-5 M $\Omega$ ) filled with $0.5 \mathrm{M}$ $\mathrm{Na}^{+}$acetate.

\section{Targeting the pre-BötC and BötC regions with microinjections in vivo}

To block fast inhibitory transmission a cocktail of $\mathrm{GABA}_{\mathrm{A}}$ (gabazine, Sigma-Aldrich) and glycine (strychnine, SigmaAldrich) receptor antagonists (in aCSF) was pressure injected $(250 \mu \mathrm{M}, 105-115 \mathrm{nl})$ into the BötC or pre-BötC bilaterally and simultaneously (1-1.5 nl/s) with small diameter $(\mathrm{OD}=15 \mu \mathrm{m}, \mathrm{ID}=7.5 \mu \mathrm{m})$ polished micropipettes (Drummond Scientific). In another set of experiments, the GABA $_{A}$ agonist muscimol was injected (100 $\left.\mu \mathrm{M}, 25-30 \mathrm{nl}\right)$ 

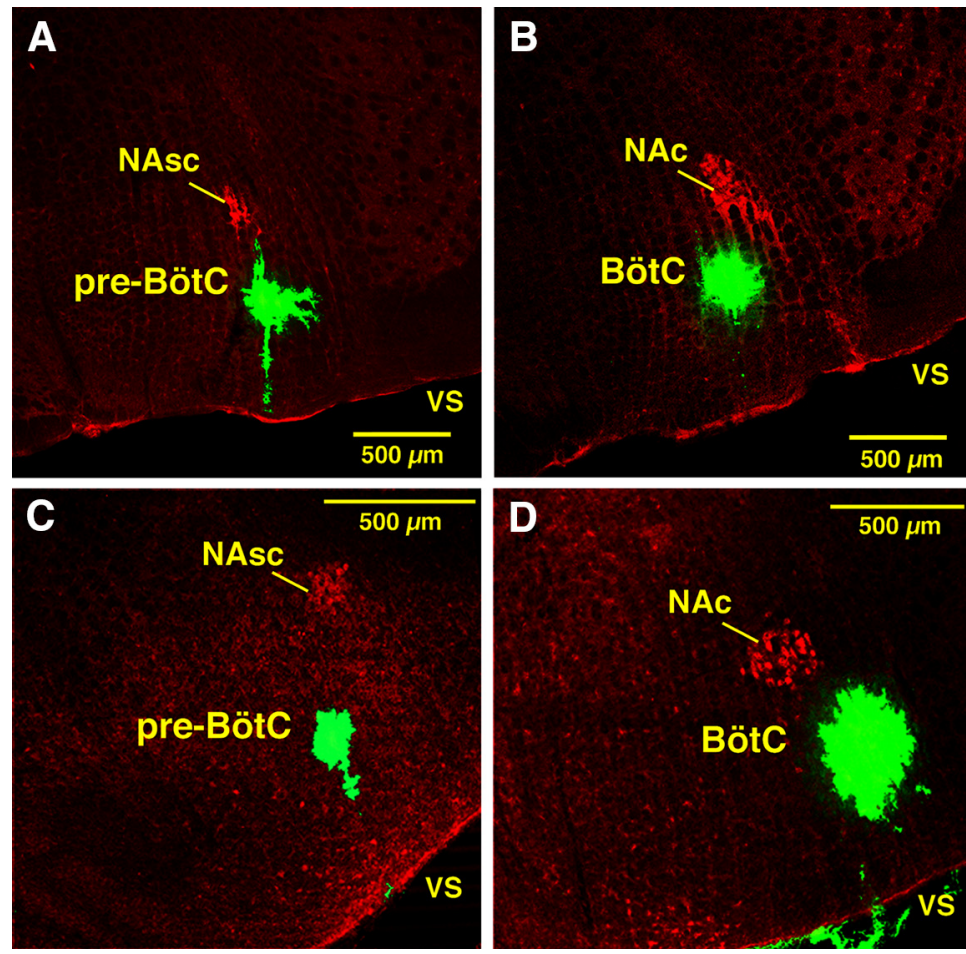

Figure 2. Confocal microscopic images of histologic sections illustrating post hoc validation of microinjection sites marked by fluorescent microspheres (green) in the pre-BötC or BötC regions in fixed sections from anesthetized adult rat in vivo preparations and juvenile rat in situ brainstem-spinal cord preparations. $\boldsymbol{A}, \boldsymbol{B}$, Coronal sections (30 $\mu \mathrm{m}$ thick) of fixed tissue at the level of pre-BötC $(\boldsymbol{A})$ and BötC $(\boldsymbol{B})$ from in vivo preparations. $\boldsymbol{C}, \boldsymbol{D}$, Coronal sections $(30 \mu \mathrm{m})$ of fixed tissue at pre-BötC $(\boldsymbol{C})$ and BötC $(\boldsymbol{D})$ levels from juvenile rat in situ brainstem-spinal cord preparations. Subdivisions of nucleus ambiguus (NAc, compact subdivision; NAsc, semi-compact subdivision), labeled with ChAT antibody (red), provide regional landmarks for pre-BötC (ventral to NAsc) and BötC (ventral to NAc) levels of the medulla. Each image is taken from serial coronal histologic sections obtained from experiments targeting these regions. Labeling with microinjected solution of fluorescent microbeads, with varying extent of local spread, indicates the approximate center of the drug microinjection sites in these examples. vs, ventral surface.

into the BötC or pre-BötC bilaterally and simultaneously. Control injections were made at the same sites with aCSF alone. All injection volumes were measured by microscopically observing the change in level of the meniscus within the micropipette. Microinjections were done after electrophysiological recording to map the extracellular single unit/neuronal population activities characteristic of the pre-BötC or BötC regions as described above, and also in some in vivo experiments after pharmacological probing by microinjection of $5 \mathrm{nl}$ of $10 \mathrm{mM}$ L-glutamate (L-Glu) to further confirm locations of the pre-BötC or BötC regions, which have site-specific responses to brief (500 ms) local L-Glu microinjections (i.e., transient apnea with increasing arterial blood pressure (ABP) for the BötC region, and tachypnea with decreasing $A B P$ for the preBötC region; see Fig. $3 C, D$ ). These blood pressure responses are typical because the pre-BötC partially overlaps with the depressor caudal ventrolateral medulla and the BötC with the pressor rostral ventrolateral medulla (RVLM; Kanjhan et al., 1995; Lipski et al., 1996; Moraes et al., 2012). According to our mapping by extracellular recordings, and consistent with characteristic perturbations obtained by our targeted L-Glu microinjections based on the neuronal activity maps, BötC and pre-BötC in isoflurane anesthetized adult male rats $(340-380 \mathrm{~g})$ occupy restricted areas: $1.8-2.1 \mathrm{~mm}$ lateral, $550-850 \mu \mathrm{m}$ depth from the ventral surface, and extending $\sim 400 \mu \mathrm{m}$ in the rostrocaudal dimension (i.e., $\sim 800-1200 \mu \mathrm{m}$ from the caudal pole of facial nucleus or $\sim 1.6-2.0 \mathrm{~mm}$ rostral to obex) for pre-BötC, and for BötC 1.9-2.2 mm lateral, $450-750 \mu \mathrm{m}$ depth, and $600-700 \mu \mathrm{m}$ in the rostrocaudal dimension $(\sim 100-750 \mu \mathrm{m}$ from the caudal pole of facial nucleus or $\sim 2.0-2.75 \mathrm{~mm}$ rostral to obex). At the end of each experiment fluorescent marker yellow-green microbeads $(0.1 \mu \mathrm{m}$ diameter, $2 \%$ solution, Invitrogen) were injected via a pipette $(\mathrm{OD}=30 \mu \mathrm{m}, \mathrm{ID}=15 \mu \mathrm{m}$; Drummond) into the site of microinjections for post hoc morphological verification (Figs. 1, 2).

Because the locations of pre-BötC and BötC in adult rats relative to the ventral surface are shallow (550-850 and $450-750 \mu \mathrm{m}$, respectively), we performed tests for back leakage of injected solution along the microinjection pipette track. Pipettes with different outer diameters $(12.5-50 \mu \mathrm{m})$ were filled with $2 \%$ of pontamine sky blue and only large diameter pipettes $(>30 \mu \mathrm{m})$ showed substantial leak of dye back to the ventral surface during microinjections. Accordingly, for drug microinjections in vivo we only used pipettes with $15 \mu \mathrm{m}$ OD that never exhibited back leak. 


\section{Microinjections in arterially perfused brainstem-spinal cord preparations in situ}

As in vivo, we bilaterally microinjected a cocktail of gabazine and strychnine and in some experiments muscimol (all drugs dissolved in the perfusate aCSF) with micropipettes $(\mathrm{ID}=20 \mu \mathrm{m})$ positioned in the pre-BötC or BötC through the ventral medullary surface with microdrives (Marzhauser) after mapping locations of these regions with extracellular recording of pre-inspiratory (pre-BötC) or expiratory (BötC) neuronal population activities. Slow, continuous microinjection was accomplished by applying low pressure $(30 \mathrm{~mm} \mathrm{Hg})$ to the pipettes with a pressure control and measurement system. In preliminary experiments we determined that, compared with the in vivo experiments, lower concentrations of drugs $(30 \mu \mathrm{M}$ of gabazine and strychnine or $10 \mu \mathrm{M}$ muscimol) bilaterally injected into either of these regions caused rapid and large disturbances of respiratory motor output and hence all experiments with the perfused brainstem-spinal cord preparations were conducted at these lower drug concentrations. In some experiments, extracellular recordings of neuronal population activity in pre-BötC (during injections in BötC) or BötC (during injections in pre-BötC) regions were made during drug microinjections. Fluorescent microbeads were microinjected to mark the sites of drug microinjection (Figs. 1, 2) as described above for the in vivo experiments.

\section{Histologic verification of microinjection sites}

Immediately after marking locations of drug microinjection sites, animals in vivo were transcardially perfused with $400 \mathrm{ml}$ of saline $\left(10-12^{\circ} \mathrm{C}, \mathrm{pH} 7.4\right)$ with heparin (1000 $\mathrm{U} / \mathrm{ml}$ ) followed by $500 \mathrm{ml}$ of $4 \%$ paraformaldehyde (wt/vol) in $0.1 \mathrm{M}$ PBS $\left(10-12^{\circ} \mathrm{C}, \mathrm{pH} 7.4\right)$. A similar procedure was followed (but with smaller volumes of saline and paraformaldehyde perfusates) for perfusion fixation of the in situ brainstem-spinal cord preparations. For both preparations, the brainstem was removed, postfixed in the same fixative for $24 \mathrm{~h}$ at $4^{\circ} \mathrm{C}$, and subsequently cryoprotected by sequential incubation in $15 \%$ and $30 \%$ sucrose in PBS and stored overnight at $4^{\circ} \mathrm{C}$ in $30 \%$ sucrose, $0.1 \mathrm{~m}$ PBS solution. The medulla oblongata was sectioned parasagittaly or coronally at 30 - or $50-\mu \mathrm{m}$-thick sections with a freezing microtome. For fluorescence immunohistochemistry, floating sections were incubated with $10 \%$ donkey serum in PBS with Triton X-100 (0.3\%) and subsequently incubated for $48-72 \mathrm{~h}$ at room temperature with primary antibodies for choline acetyltransferase (ChAT; goat antiChAT, Millipore, 1:200) to label motoneurons. Sections were then rinsed with PBS and incubated for $2 \mathrm{~h}$ with secondary antibodies for ChAT (donkey anti-goat-Dylight $488,1: 500)$. Individual sections were mounted on slides and covered with an anti-fading medium (Fluoro-Gel; Electron Microscopy Sciences). Fluorescent labeling was visualized with a laser-scanning confocal imaging system (Zeiss LSM 510).

\section{Signal analyses of respiratory parameters}

All automated analyses of respiratory parameters from digitized nerve or neuronal population activities were performed with IDL software (Exelis VIS). Inspiratory events were detected from digitally rectified and integrated phrenic nerve signals via a 200 ms window moving average and peak detection algorithm that calculated a threshold-based zero derivative (positive peak) point. Following peak detection, interburst interval (IBI; inverse of burst frequency), inspiratory time $\left(T_{1}\right)$ and expiratory time $\left(T_{E}\right)$ were measured. $T_{1}$ was measured as the original integrated burst width at $20 \%$ of the peak height above baseline; $T_{E}$ was calculated as IBI - $T_{1}$. Inspiratory amplitude (amp) was calculated by subtracting the local baseline from the peak value. The endpoint of the parameter quantification was defined when the perturbation during or after microinjections reached it maximum or the signals declined to noise level and the program started missing peak detections (which could appear as a quantum jump in $(\mathrm{BI})$.

The time courses of the changes in the above parameters for a given experimental group were variable, which we assume resulted from variability in the times required for drug diffusion to affect a sufficient number of neurons to produce the perturbations. To represent group data, we computed the mean time courses of the parameter values by the following procedures. For each experiment, the time courses of the parameters were extracted by a $30 \mathrm{~s}$ window moving median up to the defined endpoint, and the parameter values for each time series were normalized to the computed mean values during the control period (from 120 to $0 \mathrm{~s}$ before start of microinjection). Each time course was divided into 100 time points representing $1 \%$ increments from the start of microinjection (time 0 ) to the endpoint. We then computed the group mean time, and also mean values of the normalized parameters, at each of these points, which were plotted and connected by lines to represent the mean time series. The mean endpoint was plotted with its \pm SEM represented by crossbars (see Figs. $4 B-E, 6 A-D, 7 B-E, 9 A-D, 13 B-E$, and $14 D-G)$ and the SEM of the preceding normalized parameter values for the group time series were represented by a gray band. To determine statistical significance, the control values were compared with the endpoint values for each experiment within a group using a two-sided Wilcoxon signed-rank test (significant $p$ value $<0.05)$.

In addition to quantifying the respiratory parameters indicated above, we analyzed perturbations of amplitudes and durations of individual phases of the respiratory cycle (e.g., post-I activity in cVN recordings in situ) from integrated, cycle phase-triggered (peri-event) neurograms aligned at the onset of the inspiratory phase defined by PN activity. Successive cycle-triggered traces were either overlaid, or represented as a colored raster plot to depict temporal profiles of activity intensity before and during/ after drug injection periods (see Figs, 10A,B, 14B).

\section{Results}

\section{Perturbations of respiratory rhythm and pattern by targeted microinjection of I-glutamate in the pre-BötC or BötC in vivo}

As described in Materials and Methods, we microinjected L-Glu in pre-BötC and BötC regions $(n=15,10$ 
A
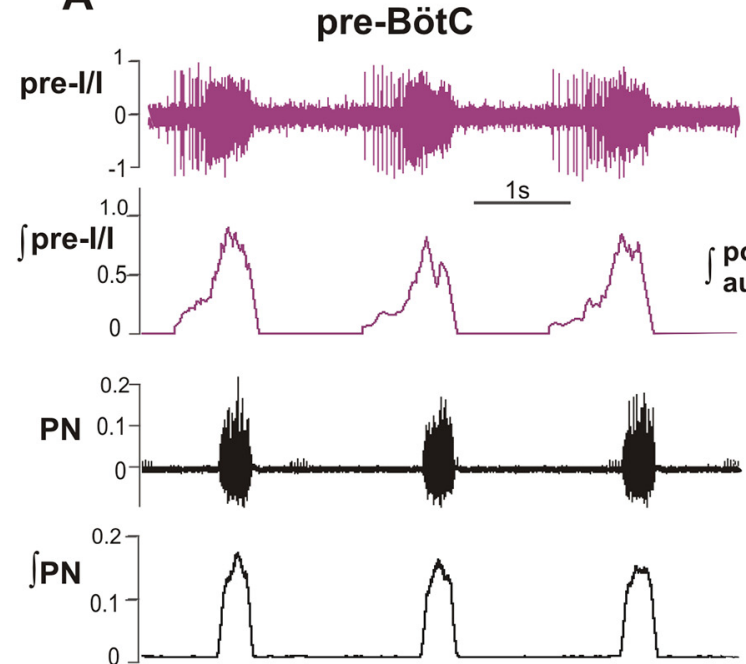

C

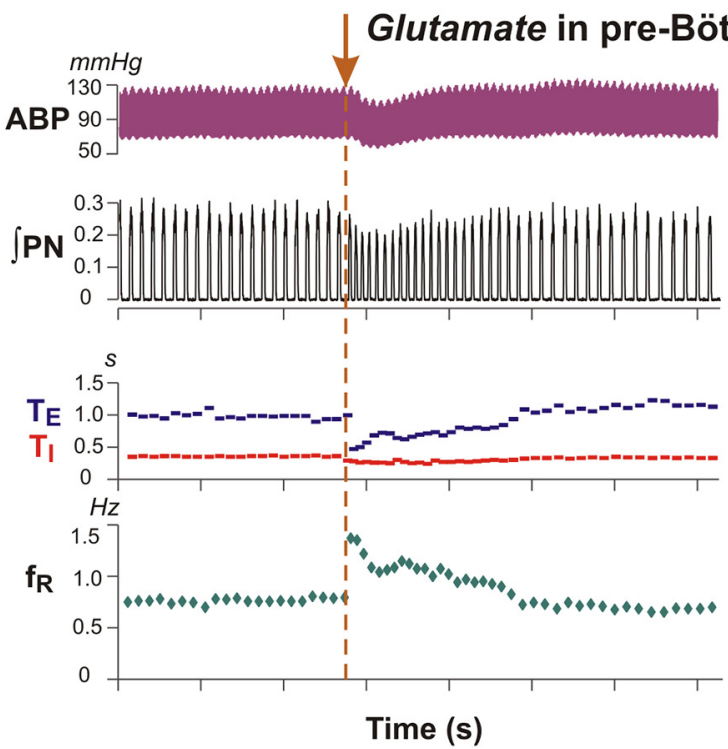

B
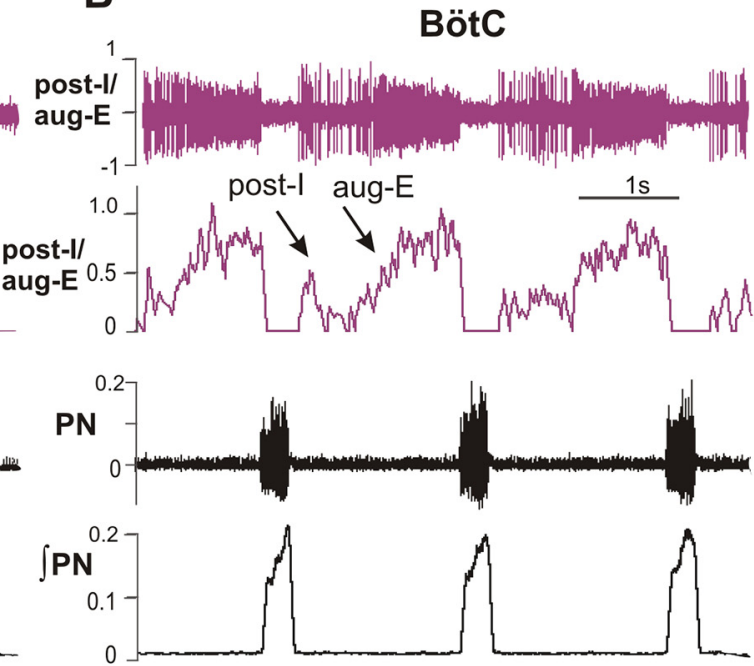

D

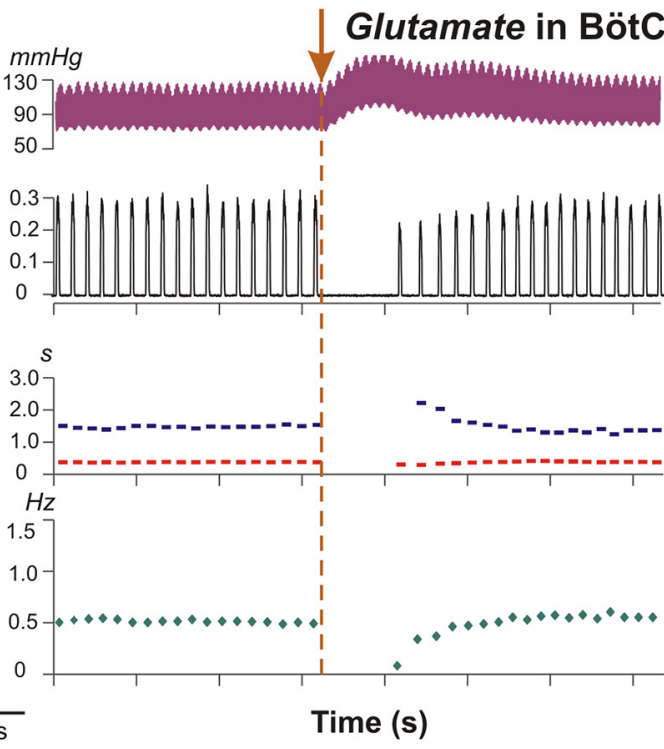

Figure 3. Characteristic profiles of extracellularly recorded neuronal population activity and examples of perturbations of inspiratory motor output activity and blood pressure produced by pharmacological excitation of neurons within the pre-BötC or BötC regions in the adult rat in vivo. A, A typical example of pre-inspiratory/inspiratory (pre-l/I) population activity used for the identification of pre-BötC. B, An example of post-I and aug-E population activity (post-I/aug-E, simultaneously recorded in this example) used for the identification of BötC. In both $\boldsymbol{A}$ and $\boldsymbol{B}$, the raw recording from the phrenic nerve (PN) and PN integrated activity ( $\mathrm{P} P \mathrm{~N})$ are shown at the bottom. C, A 500 ms duration microinjection of L-Glu (Glutamate) in the pre-BötC produced an increase in the $\int P N$ burst frequency (see the trace for integrated PN activity, $\int \mathrm{PN}$ ), and a transient decrease in the ABP. The respiratory frequency $\left(f_{\mathrm{R}}\right.$; bottom trace, green) increased primarily due to reduction in expiratory phase duration ( $T_{E}$ trace, blue) at a relatively unchanged inspiratory duration ( $T_{1}$ trace, red). $\boldsymbol{D}, A$ microinjection of $L-G l u$ in the BötC caused a rapid suppression of PN activity (see traces for $\int P N$ and inspiratory, $\mathrm{T}_{1}$, and expiratory, $\mathrm{T}_{\mathrm{E}}$, durations) accompanied by an increase of ABP. In $\boldsymbol{C}$ and $\boldsymbol{D}, \mathrm{L}-\mathrm{Glu}$ (10 mM, $5 \mathrm{nl}$ ) was microinjected bilaterally during the brief $(500 \mathrm{~ms})$ pulse; the moments of injections are indicated by brown arrows. Traces for $\mathrm{T}_{1}$, $\mathrm{T}_{\mathrm{E}}$, and $f_{\mathrm{R}}$, represent corresponding running time intervals of these parameters before, during, and recovery from L-Glu microinjection.

$\mathrm{mm}, 5 \mathrm{nl})$ to establish characteristic regional excitatory perturbations. These in vivo experiments demonstrate that activation of pre-BötC $(n=5)$ by brief $(500 \mathrm{~ms})$ L-Glu microinjections always produced a transient increase of respiratory frequency (from $28.9 \pm 4.4$ to
$44.55 \pm 6.1$ bursts $/ \mathrm{min}, p<0.0001$ ) due to a significant reduction of expiratory phase duration ( $T_{E}$; Fig. $3 C$ ). In contrast, the same L-Glu microinjections into BötC $(n=10)$ always transiently prolonged the respiratory period (by 5.4 to $21 \mathrm{~s}$ range; mean value $=10.1 \pm 4.03$ 
s; Fig. 3D), documenting site-specificity of the perturbations.

\section{Perturbations of respiratory rhythm and pattern by microinjection of gabazine and strychnine in the pre-BötC in vivo and in situ}

Microinjections of the gabazine-strychnine cocktail were performed after initially identifying the pre-BötC region electrophysiologically by recording pre-1/I neuronal population activity and/or also after microinjected L-Glu (in vivo) to identify the pre-BötC region (Fig. 3 C). Perturbations of neural activity with bilateral microinjections of $110 \mathrm{nl}$ of $250 \mu \mathrm{M}$ of gabazine and strychnine in the anesthetized adult rat in vivo were stereotypical. A representative example is shown in Fig. $\mathbf{4 A}$. Drug microinjections in pre-BötC produced an increase in the respiratory frequency $\left(f_{R}\right)$, primarily due to shortening of $T_{E}$, as well as a decrease of the amplitude of integrated PN activity. The group $(n=6)$ in vivo data are summarized in Figures $4 B-E$, which shows the mean time courses of the developing perturbations of all parameters (normalized to control values) during and after the microinjections. Perturbations developed rapidly, within $10 \mathrm{~s}$ following the onset of microinjection in all cases. The respiratory frequency increased to $184.8 \pm 5.6 \%(p=0.03), T_{1}$ decreased to $71.3 \pm 4.5 \%(p=0.03)$, and $T_{E}$ was reduced to $52.6 \pm 3.2 \%(p \leq 0.03)$ of control values. The amplitude of integrated PN activity decreased to $32.1 \pm 3.9 \%$ ( $p \leq$ 0.03 ) of pre-injection control values. These parameter values, all of which are statistically significant, were obtained at $95.83 \pm 10.03 \mathrm{~s}$ after onset of the microinjections when the perturbations approached maximum values. The average injection time for the group was $57.66 \pm 22.09 \mathrm{~s}$. After these perturbations, the time required for recovery of inspiratory activity with a pattern resembling the control activity was variable, but usually a period of minimally 40 min was required in vivo.

Similarly rapid and large perturbations were caused by bilateral microinjections of $30 \mu \mathrm{M}$ gabazine-strychnine within the pre-BötC of in situ perfused juvenile rat brainstem-spinal cord preparations $(n=6$; Fig. $5 A, B)$. Figure $5 A$ shows a typical example of the large disturbances of the respiratory frequency and amplitudes of activity recorded from $\mathrm{PN}$ and $\mathrm{CVN}$, including the pronounced increase in the frequency of PN inspiratory discharge accompanied by reductions in discharge amplitude. In the example shown in Figure $\mathbf{5 B}$, the augmented frequency of PN inspiratory discharge culminated in tonic activity, which occurred in one of six experiments. For the group data mean values of respiratory frequency increased to $175.9 \pm 18.0 \%(p=0.03), T_{1}$ slightly increased to $110.4 \pm$ $4.0 \%(p=0.03)$, and $\mathrm{T}_{E}$ decreased to $43.7 \pm 8.0 \%(p=$ 0.03 ) of control values (Fig. 6A-C). The integrated PN activity amplitude decreased to $62.3 \pm 6.0 \%(p=0.03$; Fig. 6D). These disturbances followed a similar time course to those in vivo and were measured at $84.1 \pm 17$ $s$ after the onset of microinjections (average injection time of $178.3 \mathrm{~s}$ ) when maximum changes in parameter values obtained. Recovery of control patterns of nerve activity typically occurred 15-20 min after terminating the bilateral microinjections in these in situ experiments.

\section{Disruption of respiratory rhythm and pattern by microinjection of gabazine and strychnine in the BötC in vivo and in situ}

Bilateral microinjections of gabazine-strychnine were performed in the BötC region after identifying this region by extracellular recording of augmenting expiratory and/or post-I neuronal population activity in situ and in vivo, and in some $(n=3 / 6)$ of the in vivo experiments in this group, after confirming the characteristic suppression of recorded phrenic discharge and pressor blood pressure responses by L-Glu microinjections. In contrast to the results obtained with microinjections in the pre-BötC, bilateral microinjections of $110 \mathrm{nl}$ of $250 \mu \mathrm{M}$ gabazine and strychnine into the BötC in vivo (Fig. $7 ; n=6$ ), and also the microinjections in the in situ experiments with the lower concentrations of the inhibitory receptor antagonists (Figs. 8, 9; $n=6$ ), significantly reduced the frequency of integrated $\mathrm{PN}$ discharge due primarily to a prolongation of $T_{E}$, accompanied by a reduction of $T_{1}$ and the amplitude of integrated PN activity. For the averaged group in vivo data (Fig. $7 B-E$ ), at $36.66 \pm 6.4$ s following the onset of microinjections, the respiratory frequency decreased to $25.8 \pm 8.0 \%(p \leq 0.03), T_{1}$ was reduced to $88.4 \pm 5.3 \%(p \leq 0.06)$, and $T_{E}$ increased to $257.2 \pm$ $48.0 \%(p \leq 0.03)$ of control values. The amplitude of integrated phrenic discharge decreased to $39.9 \pm 13.8 \%$ $(p \leq 0.03$ ) of control values (Fig. 7E). In two in vivo experiments, rhythmic inspiratory discharge was completely suppressed for $\geq 20 \mathrm{~s}$ (Fig. 7A). The average microinjection time for the group was $53.67 \pm 19.32 \mathrm{~s}$.

Similar perturbations occurred with bilateral microinjections of $30 \mu \mathrm{M}$ gabazine-strychnine in the BötC in situ (Figs. 8, 9). For the averaged group data $(\mathrm{n}=6$, Fig. $9 A-C)$ at $92.5 \pm 16.77 \mathrm{~s}$ after injection onset, inspiratory frequency decreased to $22.6 \pm 10.7 \%(p=0.03), T_{E}$ increased to $430.2 \pm 17.3 \%(p=0.03)$, and $T_{1}$ decreased to $56.2 \pm 13.0 \%,(p=0.03)$ of pre-injection control values. Integrated PN discharge amplitude was reduced to $15.4 \pm 7.0 \%(p=0.03$; Fig. 9D). In three of these in situ experiments, rhythmic inspiratory activity was transiently suppressed for $\geq 20 \mathrm{~s}$ (Fig. 8). These perturbations were reflected in simultaneously recorded $\mathrm{cVN}$ inspiratory activity (Fig. $8 A$ ) in all experiments analyzed and by recorded pre-BötC pre-I/l population activity (Fig. $8 B$ ) in two of these experiments, which demonstrate that the perturbations in BötC disrupt pre-BötC neuronal activity. In all experiments post-I cVN discharge was also disrupted (below) and in one experiment tonic discharge on the $\mathrm{CVN}$ was recorded during the disruption of rhythmic motor output (Fig. $\mathbf{8 A}$ ). With the bilateral injections of the inhibitory receptor antagonists in the BötC in situ or in vivo, although nerve activity could emerge after transient suppression of motor output (Figs. $7 A, 8 A$ ), such activity was disturbed relative to control activity. In all of these experiments, typically 15-25 min in situ and 40-60 min in vivo were required to recover activity resembling control patterns of motoneuronal discharge. 


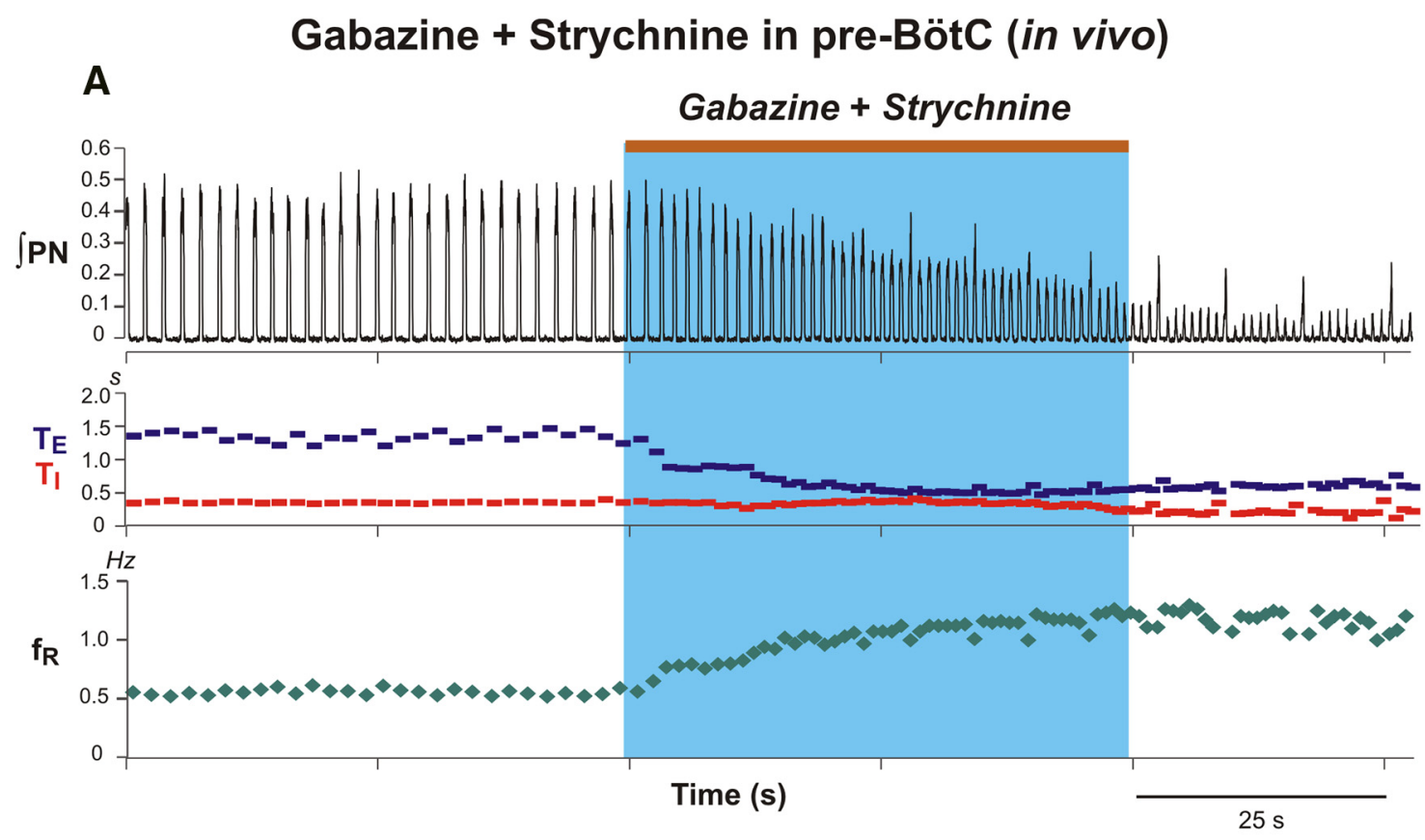

Normalized values $(n=6)$
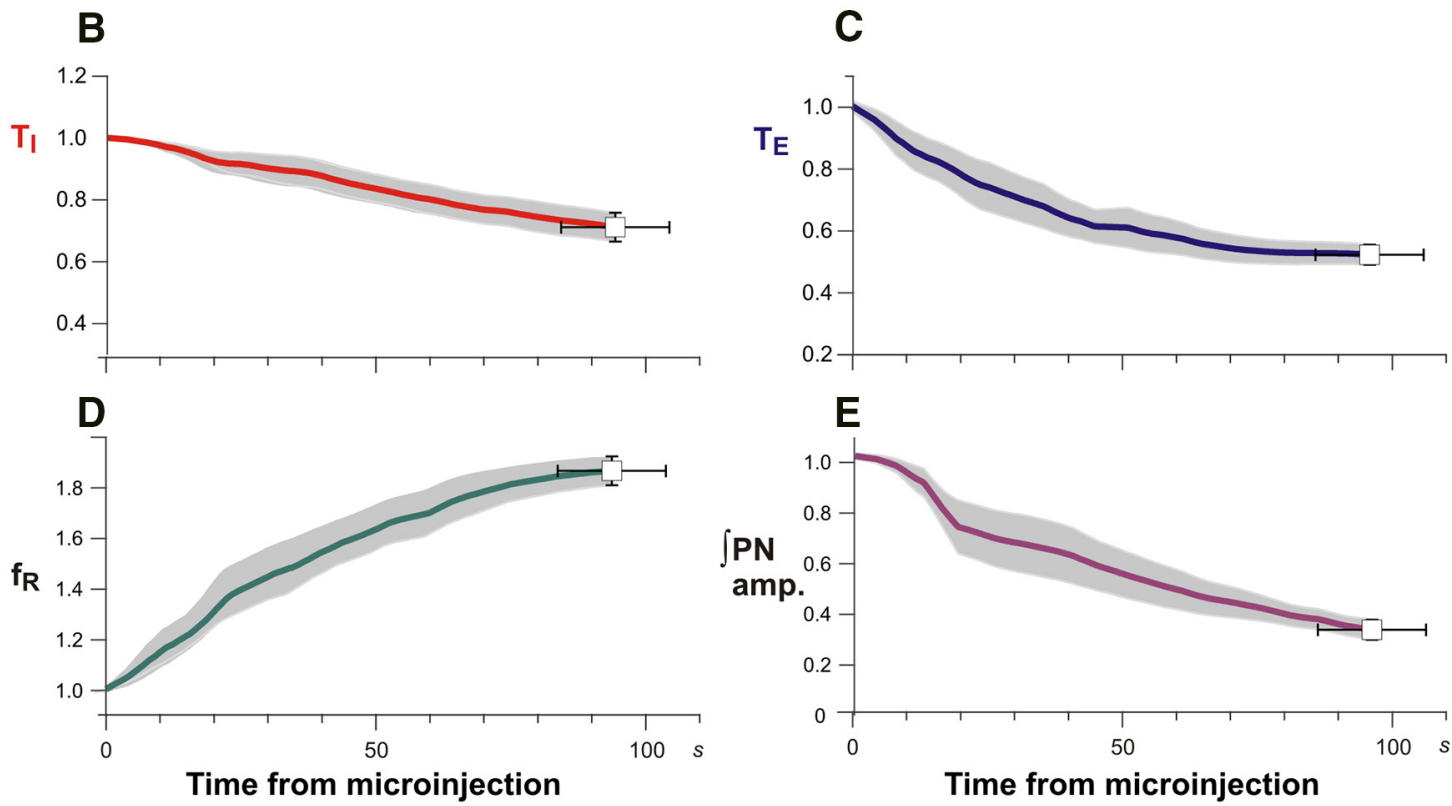

Figure 4. Perturbations of respiratory activity by pharmacologically disrupting $\mathrm{GABA}_{\mathrm{A}}$ ergic and glycinergic inhibition in the pre-BötC of anesthetized adult rat in vivo. A, Simultaneous bilateral microinjections of gabazine and strychnine (both $250 \mu \mathrm{M}$ delivered by slow microinjections of $110 \mathrm{nl}$ during the time interval indicated by the brown bar at the top and blue rectangle) caused an increase of respiratory frequency and a reduction in the integrated phrenic nerve activity amplitude ( $\int \mathrm{PN}$; black trace, top). The increase of respiratory frequency $\left(f_{\mathrm{R}}\right.$; green trace, bottom) was mainly due to reductions of expiratory phase duration ( $\mathrm{T}_{\mathrm{E}}$, blue trace) accompanied by only a small increase of inspiratory phase duration ( $T_{1}$, red trace). $\boldsymbol{B}-\boldsymbol{E}$, Group mean time series showing developing changes of normalized $T_{1}\left(\boldsymbol{B}\right.$, red $_{\mathrm{i}}$ curve), $\mathrm{T}_{\mathrm{E}}$ $\left(\boldsymbol{C}\right.$, blue curve), $f_{\mathrm{R}}\left(\boldsymbol{D}\right.$, green curve), and $\int \mathrm{PN}$ amp $(\boldsymbol{E}$, magenta curve) computed over the time window shown from the start (time $=0$ ) of microinjection. Data were computed from $\int \mathrm{PN}$ for this representative experimental group $(n=6)$. Solid colored curves are group mean time and mean normalized parameter values; gray shaded bands are \pm 1 SEM for the mean parameter values. Endpoints shown are mean time and normalized parameter values \pm 1 SEM for both at the maximal perturbation for the injection periods used.

\section{Disruption of three-phase respiratory pattern by blocking inhibition in the pre-BötC or BötC in situ}

The microinjections of gabazine-strychnine in either the pre-BötC $(n=6)$ or BötC $(n=6)$ in situ disrupted the three-phase respiratory motor output pattern as analyzed from simultaneous recordings of PN and cVN activity, the latter of which always exhibited prominent post-I discharge. Cycle-triggered averages and time-series raster 


\section{Gabazine + Strychnine in pre-BötC (in situ)}

A

\section{Gabazine + Strychnine}

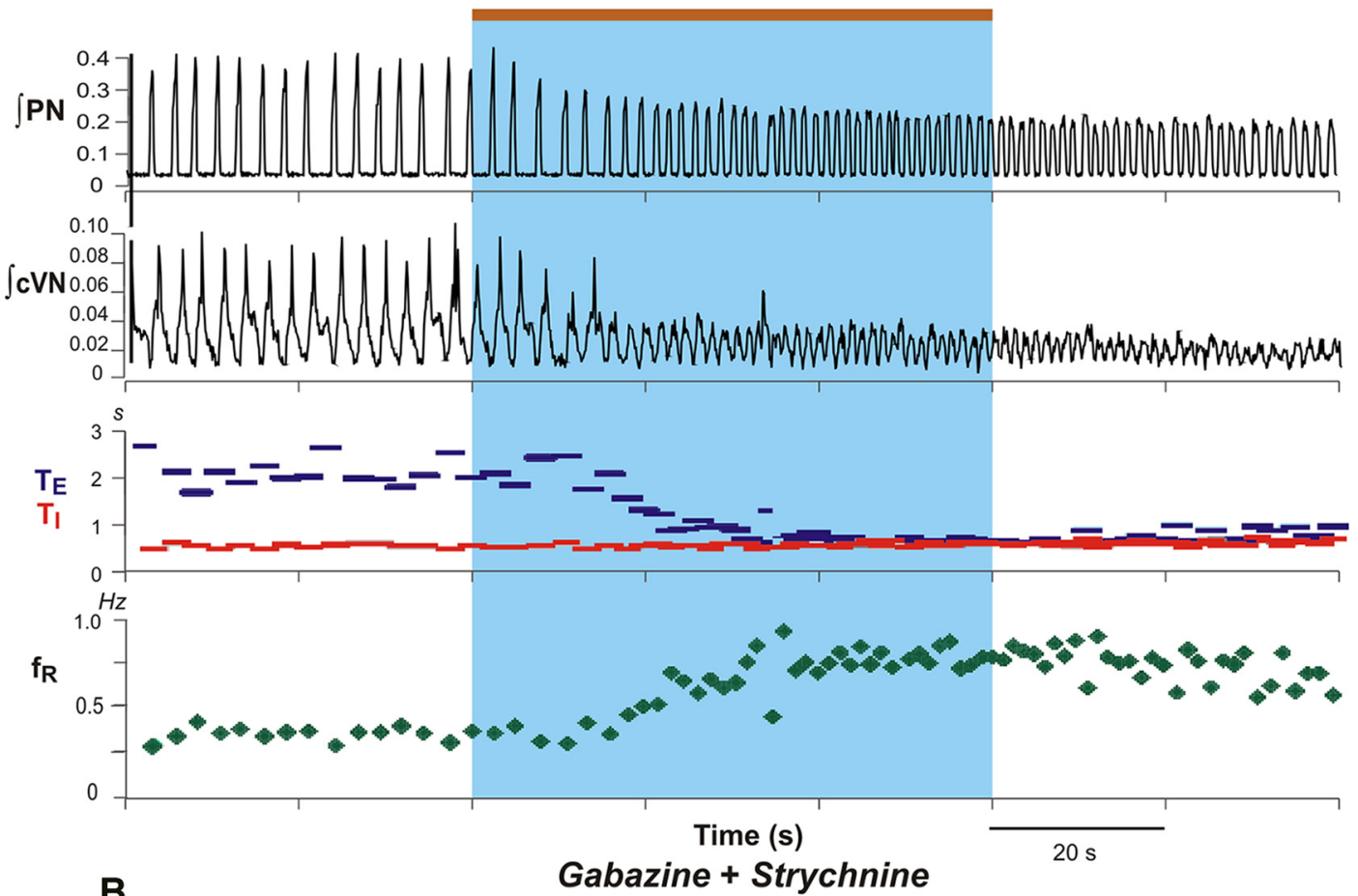

B

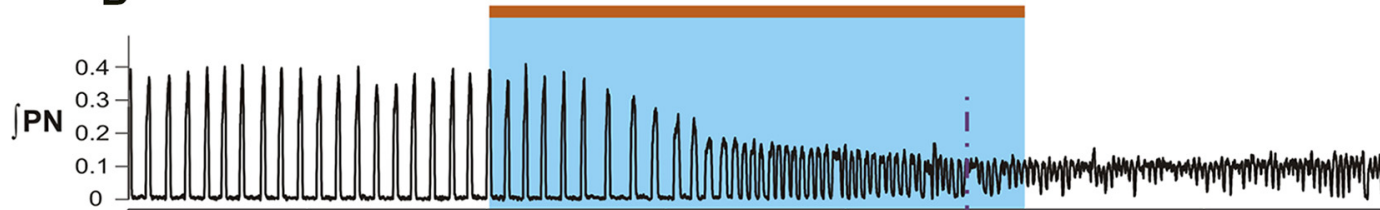

$\int \mathrm{cVN}_{0}^{0 .}$

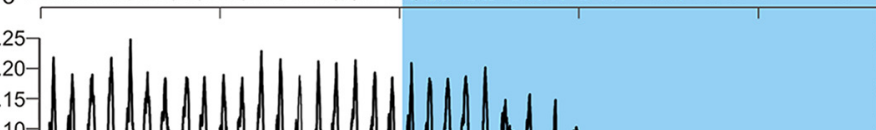

$0.05-12 \sqrt{ }$. W.

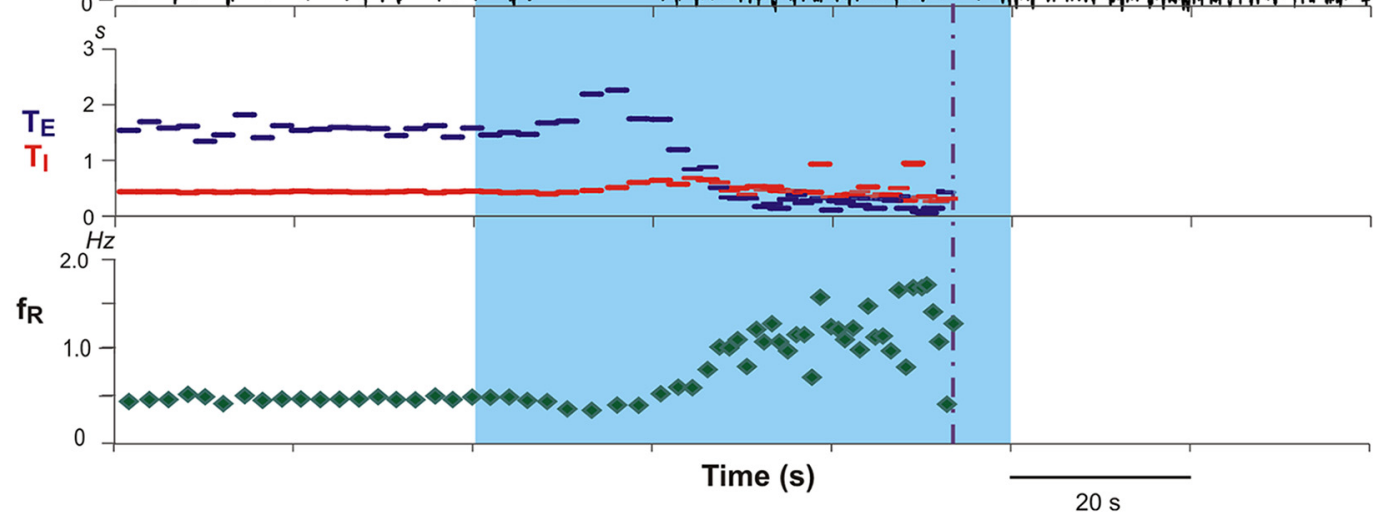

Figure 5. Perturbations of respiratory activity by pharmacologically disrupting $\mathrm{GABA}_{\mathrm{A}}$ ergic and glycinergic inhibition in the pre-BötC of juvenile rat perfused brainstem-spinal cord in situ. $\boldsymbol{A}$, Example of experimental recordings illustrating perturbations of integrated $\mathrm{PN}\left(\int \mathrm{PN}\right)$ and $\mathrm{cVN}\left(\int \mathrm{cVN}\right)$ activities by simultaneous bilateral microinjections of gabazine and strychnine (both $30 \mu \mathrm{M}$; injection period indicated by the brown bar at the top and blue rectangle), which caused an increase of respiratory frequency $\left(f_{\mathrm{R}}\right)$ due to a reduction in $T_{E}$ (blue) without significant changes in $T_{1}$, and a reduction in amplitude of $\int P N$ and $\int c V N$. $B$, Example of perturbations where the $\int P N$ activity progressed to tonic activity, as indicated by the upward shift of the $\int \mathrm{PN}$ signal baseline. Analysis of respiratory parameters in this case was performed up to the time point indicated by the vertical dot-dashed line. The progressive reduction in $\int \mathrm{cVN}$ amplitude in both examples reflects in part a reduction and eventual loss of post-I activity (Fig. 10 shows a more detailed analysis). 


\section{Gabazine + Strychnine in pre-BötC (in situ)}

Normalized values $(n=6)$
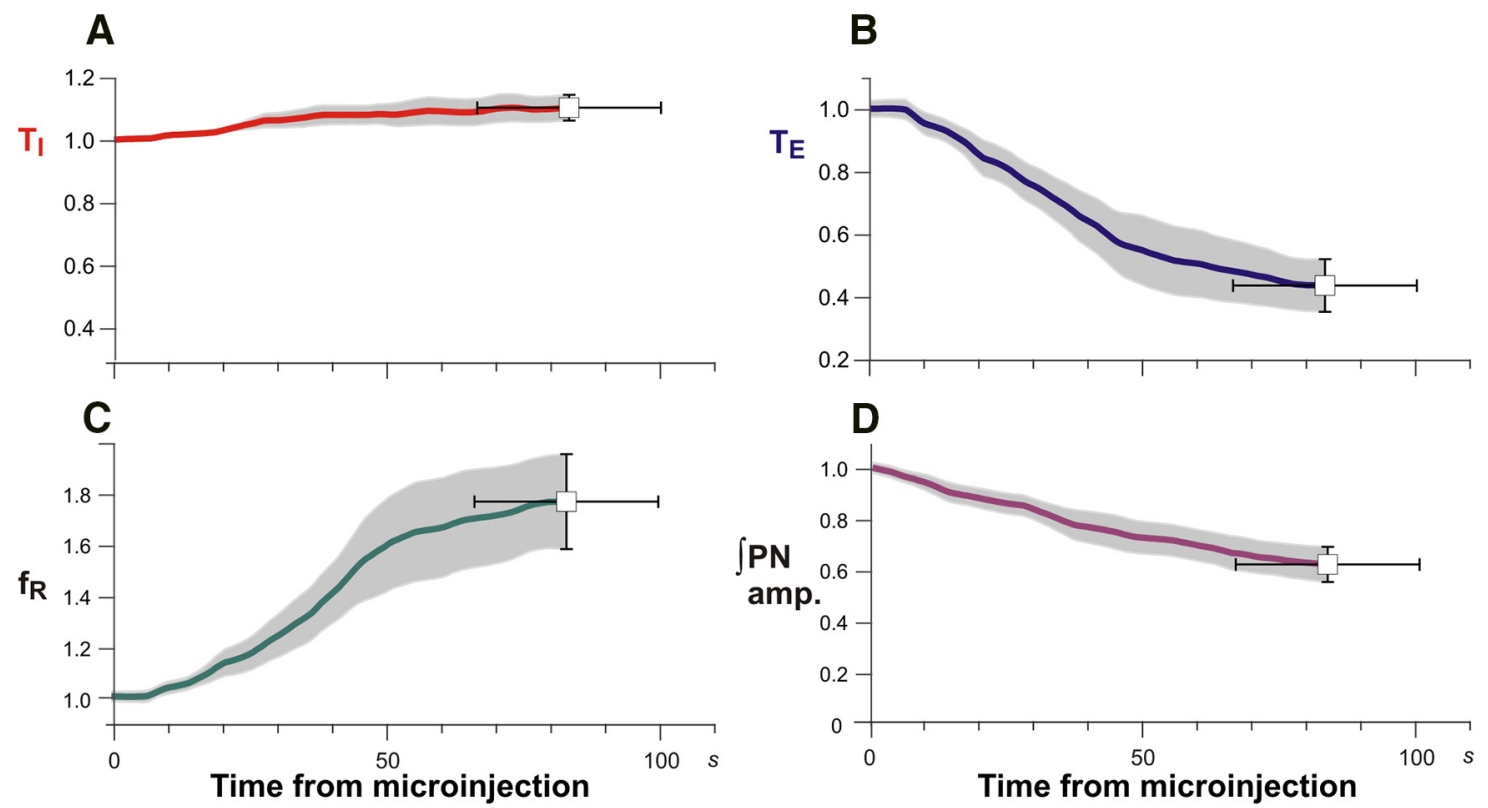

Figure 6. Group data summarizing changes of respiratory activity parameters by pharmacologically disrupting $\mathrm{GABA}_{A} \mathrm{ergic}_{\text {and }}$ glycinergic inhibition in the pre-BötC of juvenile rat perfused brainstem-spinal cord in situ. $\boldsymbol{A}-\boldsymbol{D}$, Mean time series from the start of microinjection showing developing changes of normalized $T_{1}\left(\boldsymbol{A}\right.$, red curve), $\mathrm{T}_{\mathrm{E}}$ (B, blue curve), respiratory frequency $\left(f_{\mathrm{B}} ; \boldsymbol{C}\right.$, green curve), and integrated PN discharge amplitude ( $\boldsymbol{D}$, amp, magenta curve), which were computed from integrated phrenic nerve activity for a representative experimental group $(n=6)$. Solid colored curves are group mean time and normalized parameter values; gray bands are \pm 1 SEM for the mean normalized parameter values as in Figure $4 B-E$.

plots of integrated $\mathrm{PN}$ and $\mathrm{cVN}$ shown in Figure $10 A, B$ illustrate that $\mathrm{cVN}$ post-I activity is eliminated as $\mathrm{GABA}_{\mathrm{A}}$ ergic and glycinergic inhibition is attenuated in either region. This loss of post-I activity as the druginduced perturbation develops represents a transformation from a three-phase to a two-phase motor output pattern (Smith et al., 2007).

\section{Site-specific perturbations of rhythm and pattern by microinjections of muscimol in the pre-BötC and BötC in vivo and in situ}

The differential perturbations of inspiratory discharge frequency with the $\mathrm{GABA}_{\mathrm{A}}$ and glycine receptor antagonists in the BötC (frequency decrease) versus the pre-BötC (frequency increase) demonstrate site-specificity of the perturbations. To further test for site-specific perturbations by manipulating local inhibition, we regionally inhibited/attenuated neuronal activity by bilateral microinjection of the $\mathrm{GABA}_{A}$ receptor agonist muscimol. In these experiments, the pre-BötC and BötC regions were first identified electrophysiologically by extracellular recording and/or by microinjection of L-Glu in vivo. As illustrated in Figure 11, bilateral microinjection of 25-30 nl of $100 \mu \mathrm{M}$ muscimol in the pre-BötC in vivo progressively reduced the frequency and amplitude of phrenic discharge and eliminated inspiratory motor output within 20 $s$ in all experiments $(n=7)$. The suppression of inspiratory activity persisted for $40.1 \pm 26.8 \mathrm{~s}$ during which rhythmic inspiratory activity could be restored immediately by subsequent bilateral microinjection of $110 \mathrm{nl}$ of $250 \mu \mathrm{M}$ gabazine at the same site (Fig. 11). This latter result also demonstrated the efficacy of gabazine at the concentrations used in our experiments to antagonize activation of $\mathrm{GABA}_{A}$ receptors in a regionally specific manner. Bilateral microinjections of lower concentrations of muscimol $(10 \mu \mathrm{M})$ in the pre-BötC in the in situ preparations $(n=5)$ also rapidly suppressed phrenic inspiratory activity (Fig. 12).

In experiments targeting the BötC in vivo $(n=7)$, bilateral microinjection of the same volume and concentration of muscimol progressively increased discharge frequency, due primarily to a reduction of $T_{E}$, and reduced the amplitude of integrated phrenic inspiratory discharge (Fig. 13). For the averaged group in vivo data at $486.4 \pm$ $67.6 \mathrm{~s}$ after injection onset, integrated phrenic discharge amplitude was reduced to $58.9 \pm 8.9 \%(\rho=0.015)$, inspiratory frequency increased to $175.19 \pm 8.4 \%$ $(p=0.015), T_{E}$ decreased to $51.4 \pm 3.3 \%(p=0.015)$, and $T_{1}$ decreased to $83.4 \pm 3.3 \%(p=0.015)$ of pre-injection control values (Fig. 13B-D). Comparable perturbations were obtained with bilateral microinjections of $10 \mu \mathrm{M}$ muscimol in the in situ preparations (Fig. 14; $n=6$ ). At $139 \pm$ $20 \mathrm{~s}$ following the onset of the microinjections, the group averaged inspiratory frequency increased to $148.1 \pm$ 


\section{Gabazine + Strychnine in BötC (in vivo)}

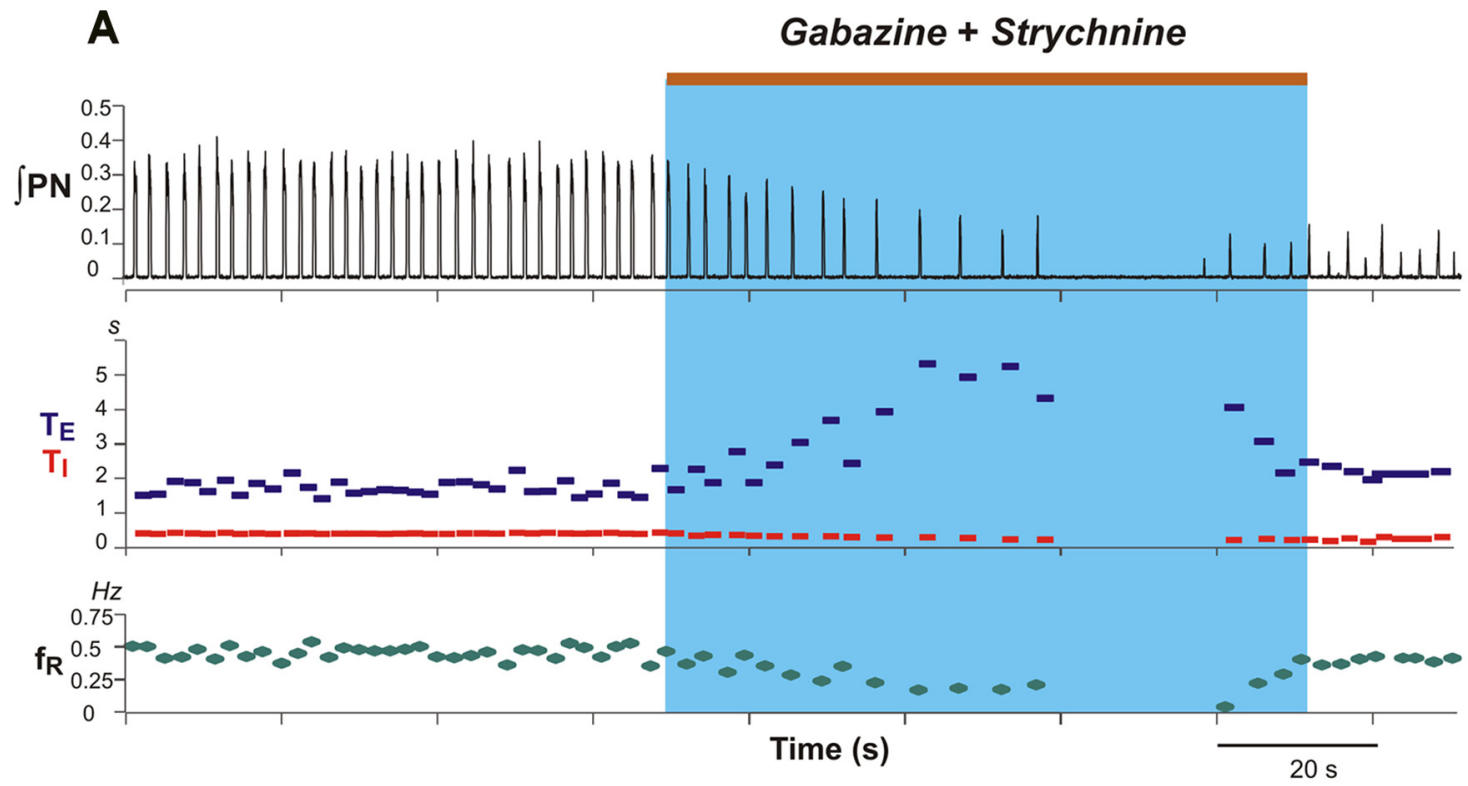

Normalized values $(n=6)$

B

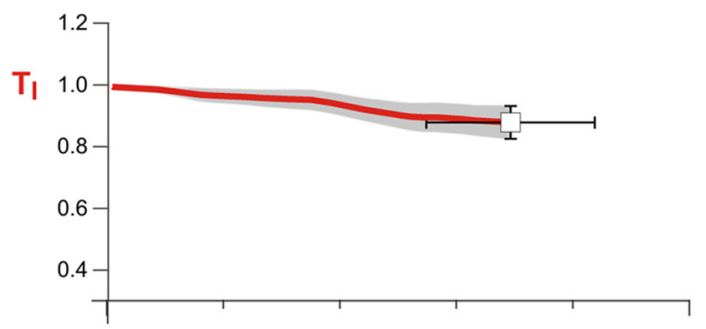

D

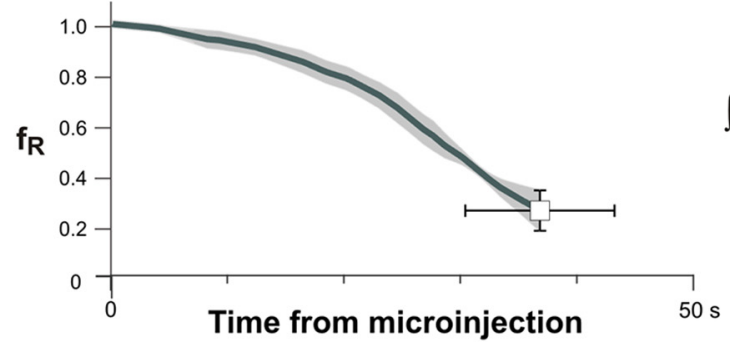

C
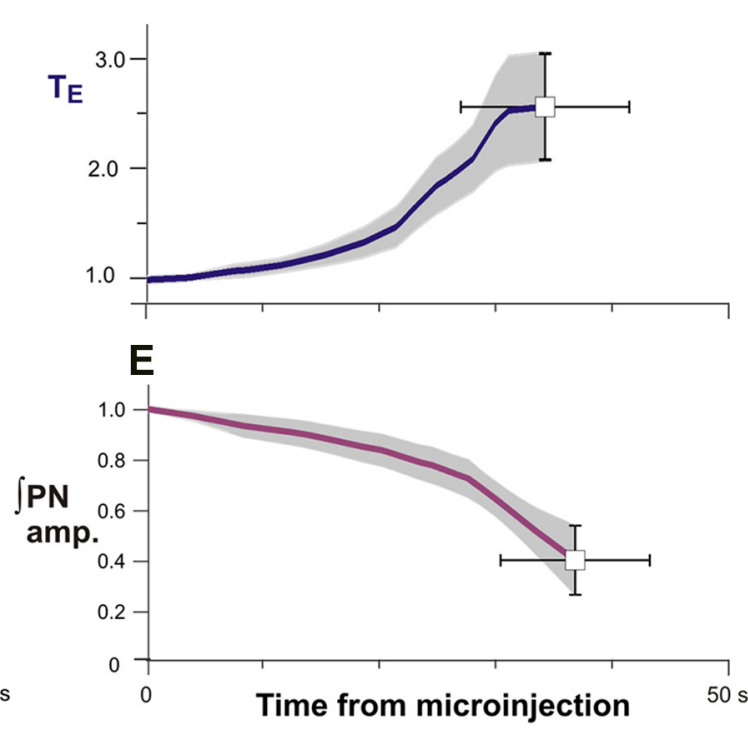

Figure 7. Perturbations of respiratory activity by pharmacologically disrupting of $\mathrm{GABA}_{\mathrm{A}}$ ergic and glycinergic inhibition in the BötC of adult rat in vivo. $\boldsymbol{A}$, Gabazine and strychnine cocktail $(110 \mathrm{nl}, 250 \mu \mathrm{m})$ slowly injected during time period indicated (by the brown bar at the top and blue rectangle), progressively reduced respiratory frequency leading to transient apnea in this example ( $\int P N$, black trace, top). Changes in the inspiratory $\left(\mathrm{T}_{1}\right.$, red) and expiratory $\left(\mathrm{T}_{\mathrm{E}}\right.$, blue) phase durations and respiratory frequency $\left(f_{\mathrm{R}}\right)$ are shown at the bottom; $f_{\mathrm{R}}$ was reduced mainly due to prolongation of $\mathrm{T}_{\mathrm{E}}$. $\boldsymbol{B}-\boldsymbol{E}$, Mean time series from the start of microinjection showing developing changes of normalized $T_{1}\left(\boldsymbol{B}\right.$, red curve), $\mathrm{T}_{E}\left(\boldsymbol{C}\right.$, blue curve), $f_{\mathrm{R}}(\boldsymbol{D}$, green curve), and integrated $\mathrm{PN}$ activity amplitude $(\boldsymbol{E}$, magenta curve) for a representative experimental group $(n=6)$. Solid colored curves are group mean time and normalized parameter values; gray bands are \pm 1 SEM for the mean normalized parameter values.

$16.88 \%(p=0.03), T_{E}$ decreased to $53.1 \pm 8.0 \%(p=$ $0.03)$, and $T_{1}$ was essentially unchanged $(99.0 \pm 15.3 \%$ of pre-injection values, $p=1.0$; Fig. 14D-F). The integrated phrenic discharge amplitude was reduced to $75.4 \pm 6.4 \%$ $(p=0.03$; Fig. 14G). In addition, integrated cVN post-I activity was progressively reduced and eliminated by muscimol in the BötC in situ (Fig. 14B).

\section{Discussion}

Role of synaptic inhibition within and between preBötC and BötC for respiratory rhythm and pattern generation

For the discussion of the role of synaptic inhibition in respiratory rhythm and pattern generation, it is necessary 


\title{
Gabazine + Strychnine in BötC (in situ)
}

A

\author{
Gabazine + Strychnine
}

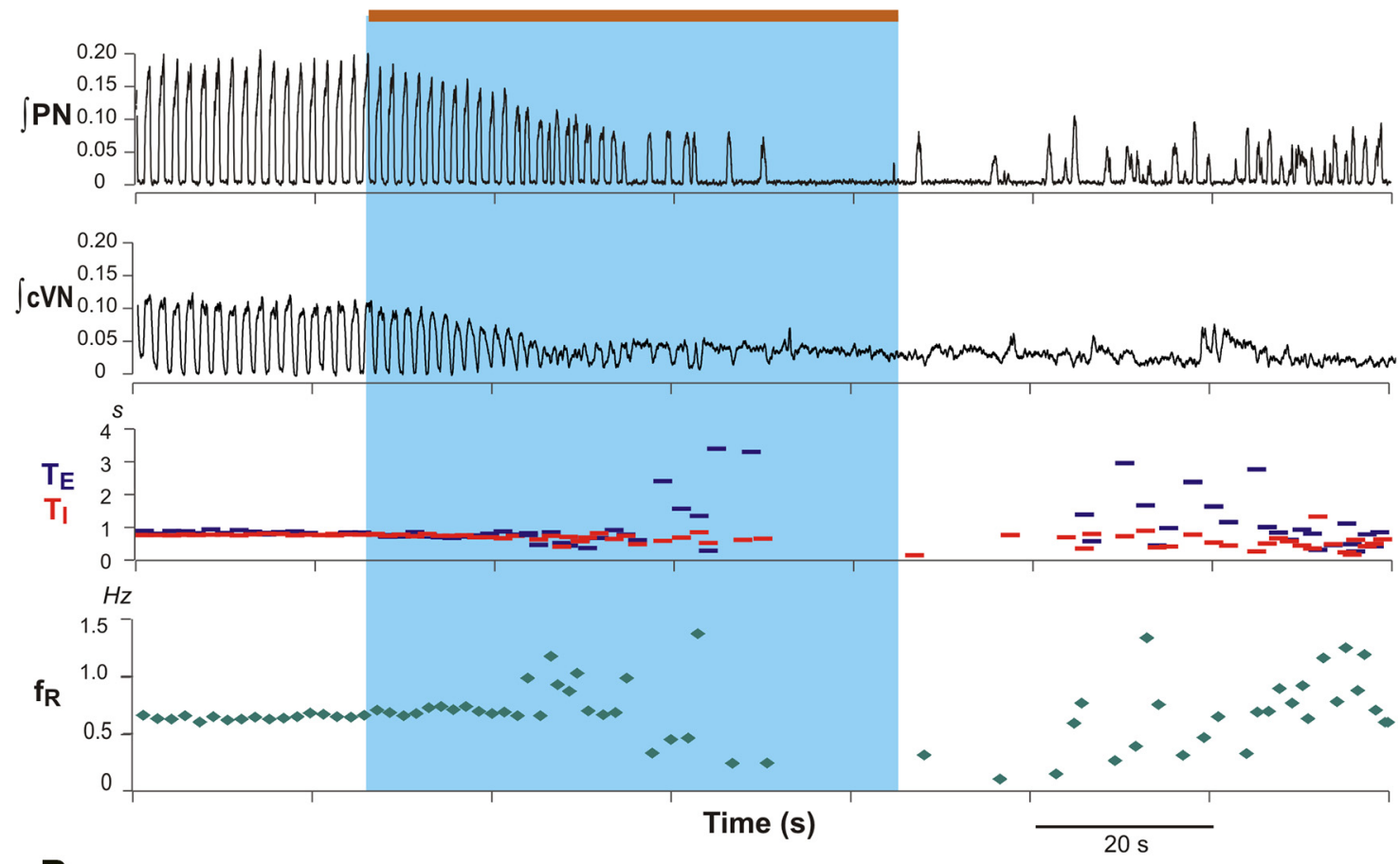

B

Gabazine + Strychnine
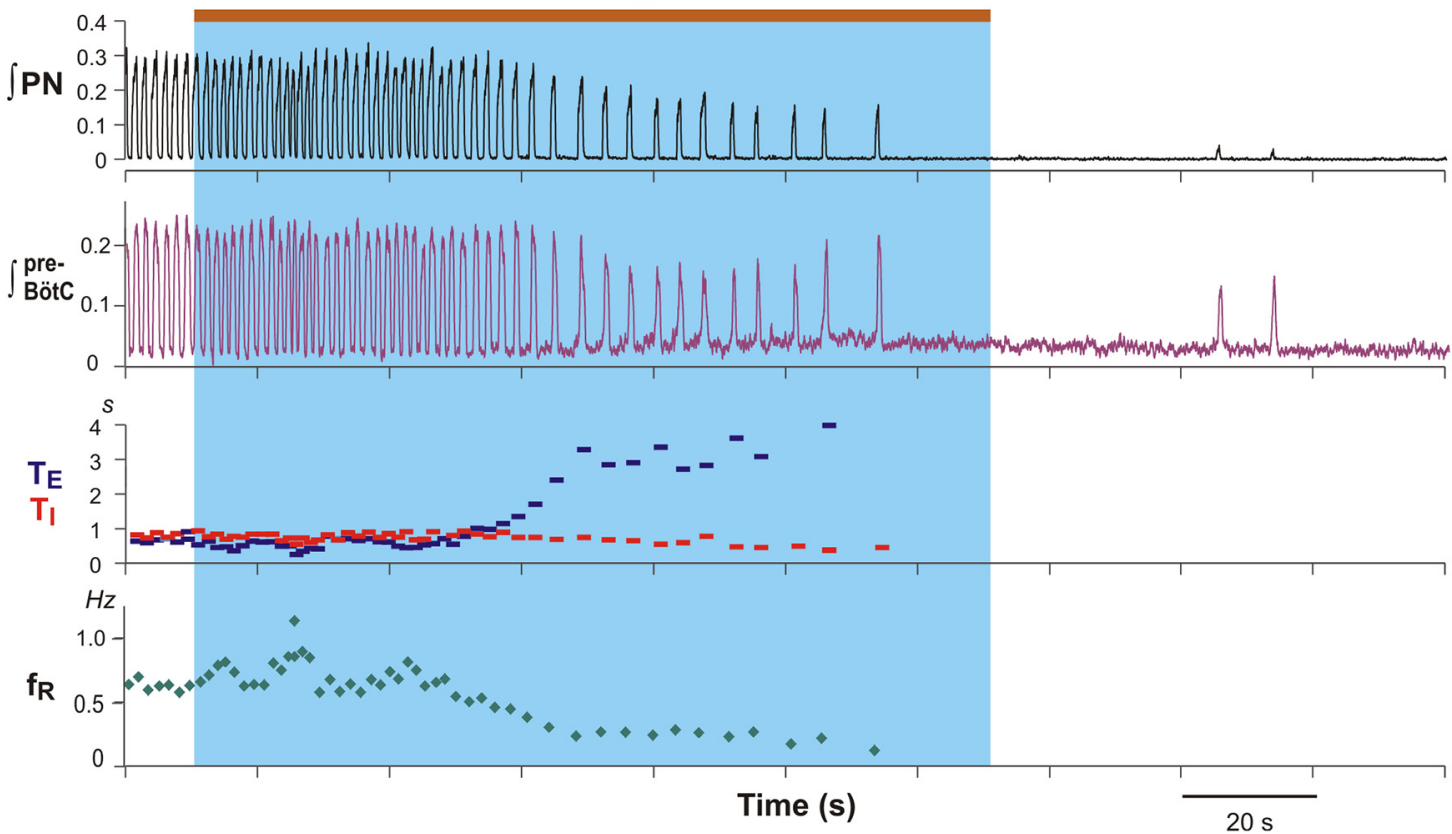

Figure 8. Disruption of rhythmic respiratory activity by block/attenuation of GABA $\mathrm{ergic}_{\mathrm{A}}$ and glycinergic inhibition in the BötC of in situ perfused brainstem-spinal cord preparations. $\boldsymbol{A}, \boldsymbol{B}$, Two examples from experiments using different preparations illustrating perturbations caused by microinjections of gabazine and strychnine (30 $\mu \mathrm{m}$ slowly injected during the period indicated by the brown bars at the top and blue rectangles). As in the vivo experiments, respiratory frequency was reduced by a prolonged $\mathrm{T}_{\mathrm{E}}$, integrated phrenic nerve discharge amplitude ( $\left.\int \mathrm{PN}\right)$ was also reduced, and ultimately apnea occurred. Simultaneously recorded integrated cVN $\left(\int \mathrm{cVN}\right)$ in $\boldsymbol{A}$ shows disruption of rhythmic activity and tonic discharge (shift of integrated activity baseline) during apneic period. $\boldsymbol{B}$, Simultaneously recorded integrated pre-BötC pre-l/I population activity from another experiment also reflects the reduction of inspiratory frequency and termination of rhythmic activity during bilateral microinjections of the inhibitory receptor blockers in BötC. 


\section{Gabazine + Strychnine in BötC (in situ)}

\section{Normalized values $(n=6)$}
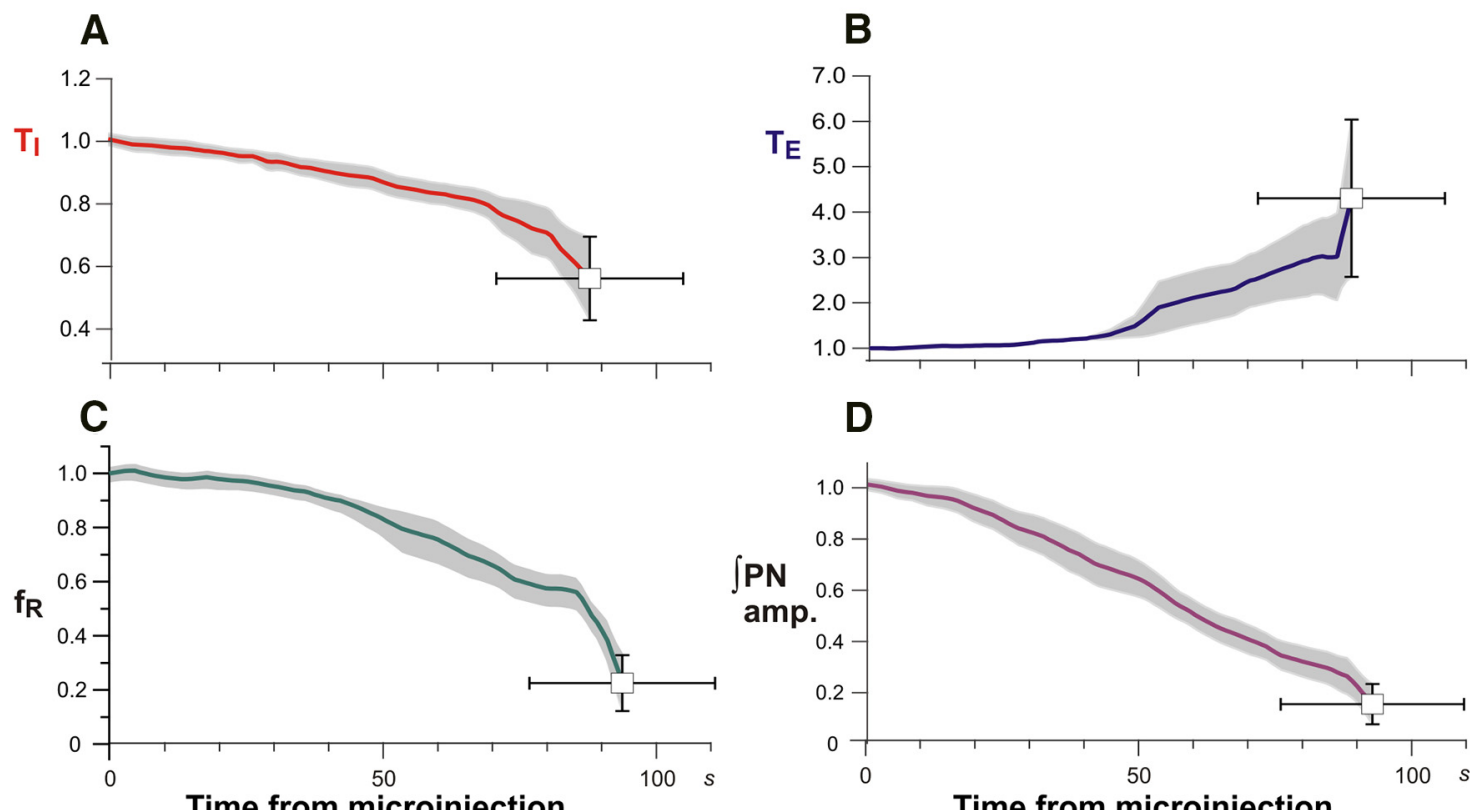

Figure 9. Group data $(n=6)$ summarizing perturbations of respiratory rhythm and motor output pattern parameters by disrupting GABA $_{A}$ ergic and glycinergic inhibition in the BötC of in situ perfused brainstem-spinal cord preparations. Solid colored curves in these time series are mean time and normalized parameter values and gray bands are $\pm 1 \mathrm{SEM}$ for the normalized values of $T_{1}(\boldsymbol{A})$, $T_{E}(\boldsymbol{B})$, respiratory frequency $\left(f_{\mathrm{R}} ; \boldsymbol{C}\right)$, and integrated inspiratory activity amplitude $(\boldsymbol{D})$ computed from the start of microinjections from recordings of integrated $\mathrm{PN}$ activity.

to clarify our definitions of the terms "rhythm" and "pattern". We define rhythm as the respiratory cycle period/ frequency. Respiratory pattern is defined as all features of activity occurring within the respiratory cycle, including the presence and coordination of the different activity phases (inspiratory, post-inspiratory, late expiratory) as recorded from neuronal populations/nerve motor output activity, as well as the durations, amplitudes, and shapes of integrated activity within phases.

Brainstem respiratory networks consist of excitatory and inhibitory circuits distributed within interacting regions of the medulla and pons including the medullary pre-BötC and BötC, which are proposed to contain core circuits involved in respiratory rhythm and pattern generation. Although there is general agreement that excitatory pre-BötC circuits generate rhythmic inspiratory activity transmitted to and driving spinal and cranial inspiratory motor outputs, functional roles of inhibitory pre-BötC and BötC circuits continue to be debated (Smith et al., 2009, 2013, cf. Feldman et al., 2013; Janczewski et al., 2013; Richter and Smith, 2014). It has been postulated that phasic synaptic inhibition originating in these circuits, known to contain populations of rhythmically active glycinergic and GABAergic neurons, are critically involved in generating the three-phase pattern of respiratory neuron activity during normal breathing by shaping firing patterns of active populations of neurons, orchestrating phase transitions, and controlling which populations are inactive during each phase (Rybak et al., 2004, 2007; Smith et al., 2007, 2009,2013). Inspiratory and expiratory neurons in these regions receive phasic volleys of inhibitory post-synaptic potentials/currents as clearly established by intracellular recordings (Schmid et al., 1996; Molkov et al., 2012; Shevtsova et al., 2014; Richter and Smith, 2014), and the inhibitory neurons in the pre-BötC and BötC are proposed to interact during the respiratory cycle via mutual inhibitory connections for dynamic control of rhythm generation, although these interactions have not been definitely established experimentally.

We have further evaluated roles of the glycinergic and GABAergic synaptic inhibition within the pre-BötC and BötC by regionally disrupting/attenuating this inhibition pharmacologically with specific receptor antagonists, which has been attempted in previous studies in several species (cat, rat, rabbit) in vivo, most recently in adult rats with conflicting results (Janczewski et al., 2013). In the present experiments we have established that pharmacologically attenuating $\mathrm{GABA}_{\mathrm{A}}$ and glycine receptormediated inhibition in the pre-BötC or BötC causes major site-specific perturbations of respiratory rhythm and pattern both in anesthetized, vagotomized adult rats in vivo and unanaesthetized, vagotomized juvenile rat perfused brainstem-spinal cord preparations in situ. The results obtained in vivo and in situ are congruent and confirm that: 


\section{A Gabazine + Strychnine in pre-BötC (in situ)}

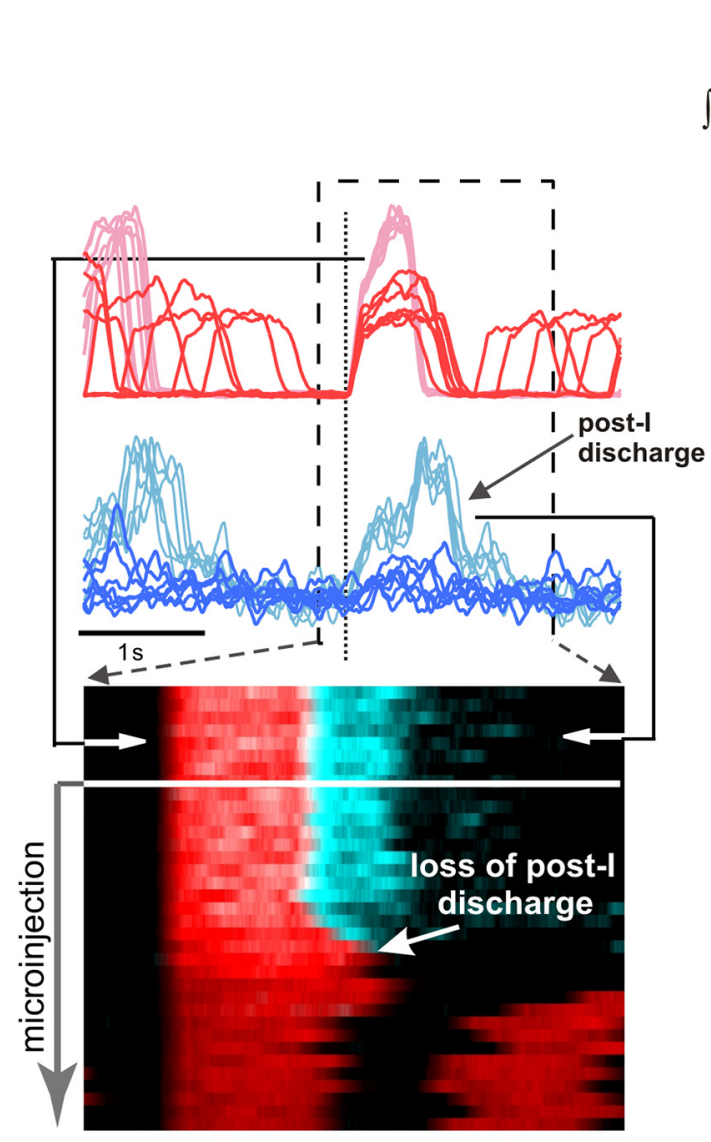

\section{B Gabazine + Strychnine in BötC (in situ)}

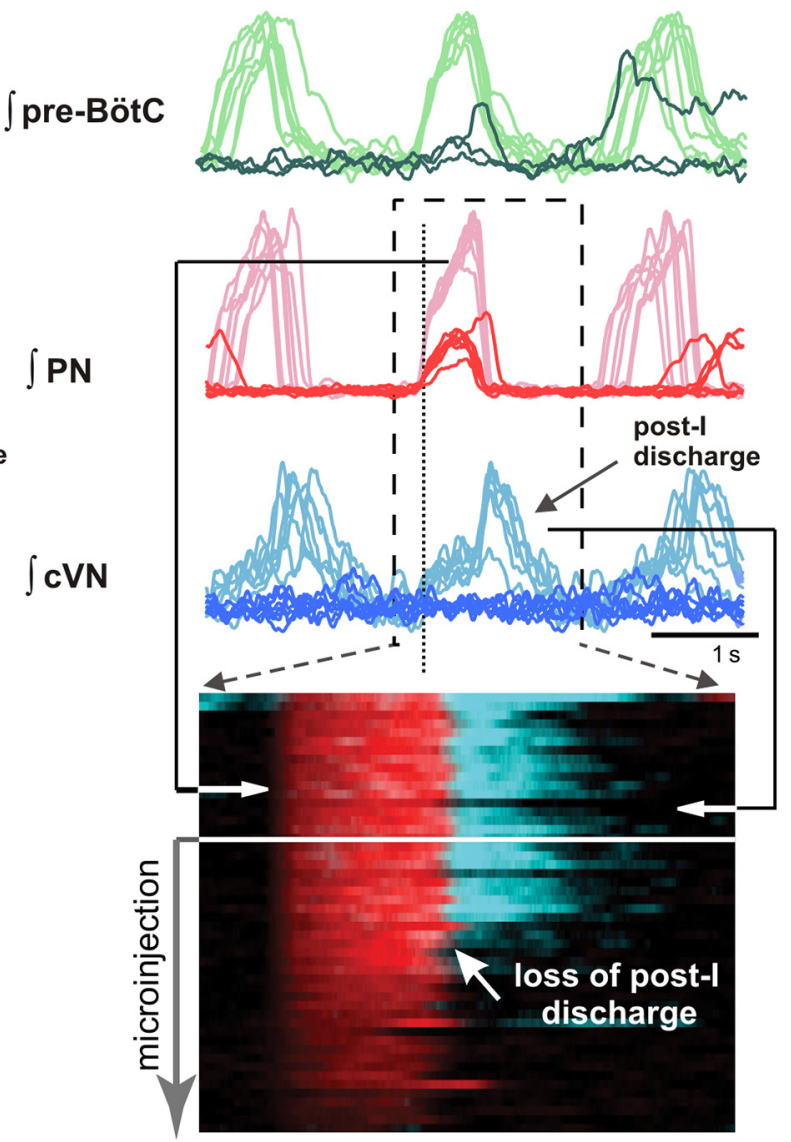

Figure 10. Disturbances of three-phase respiratory pattern including disruption of post-I activity by bilaterally microinjected gabazine and strychnine (30 $\mu \mathrm{m}$ each) in the pre-BötC $(\boldsymbol{A})$ or BötC $(\boldsymbol{B})$ of in situ perfused juvenile rat brainstem-spinal cord preparations. Upper traces in $\boldsymbol{A}$ and $\boldsymbol{B}$ show seven consecutive overlaid and aligned integrated PN (red traces) and cVN (blue traces) activity signals in the pre-microinjection control period (light red and light blue larger amplitude traces, respectively) and also seven overlaid traces of recorded signals during microinjections (dark red and dark blue traces with reduced amplitudes). Simultaneously recorded pre-BötC pre-I/I population activity before and during microinjections (light and dark green traces, respectively) is also shown at the top in $\boldsymbol{B}$ to indicate activity perturbations in this region occurring with disruption of inhibition in BötC. Signals are aligned (cycle-triggered) at the onset of PN inspiratory activity indicated by the vertical dotted line in $\boldsymbol{A}$ and $\boldsymbol{B}$. Raster plots below overlaid traces show consecutive series of inspiratory-onset aligned respiratory cycles with integrated PN inspiratory (red) and cVN including post-I (blue) activities before and during microinjections. Arrows on the raster plots indicate time interval over which the overlaid traces above the raster plots were obtained for the pre-microinjection period. During microinjections in the pre-BötC or BötC, the amplitude of inspiratory and post-I activity is progressively reduced, and post-I discharge is eliminated as seen on the aligned (darker) $\int c V N$ traces above the raster plots and in the raster plots with loss of post-I discharge indicated (white arrow).

(1) Blocking inhibition in the pre-BötC augmented inspiratory discharge frequency due to a shortening of $T_{E}$, reduced inspiratory discharge amplitude, and eliminated post-I activity thereby disrupting the normal three-phase respiratory pattern. In some cases, rhythmic motor output was completely terminated.

(2) Blocking inhibition in the BötC reduced inspiratory discharge frequency associated with a lengthened $T_{E}$, reduced the amplitude of inspiratory discharge, disrupted the three-phase pattern due to a loss of rhythmic post-I activity, and in some cases transiently terminated inspiratory motor output accompanying a loss of pre-BötC inspiratory activity.
Therefore, we conclude that ongoing glycinergic and $\mathrm{GABA}_{\mathrm{A}}$ ergic inhibition in pre-BötC and BötC circuits are fundamentally involved in dynamical regulation of respiratory rhythm generation and are required for generating the normal three-phase respiratory pattern. Our studies also confirm a fundamental role of BötC inhibitory circuits and their interactions with pre-BötC inspiratory rhythm generating circuits.

\section{Comparisons with results from previous targeted pharmacology studies}

Our results, obtained in juvenile and adult rats, are consistent with previous studies in anesthetized, non- 


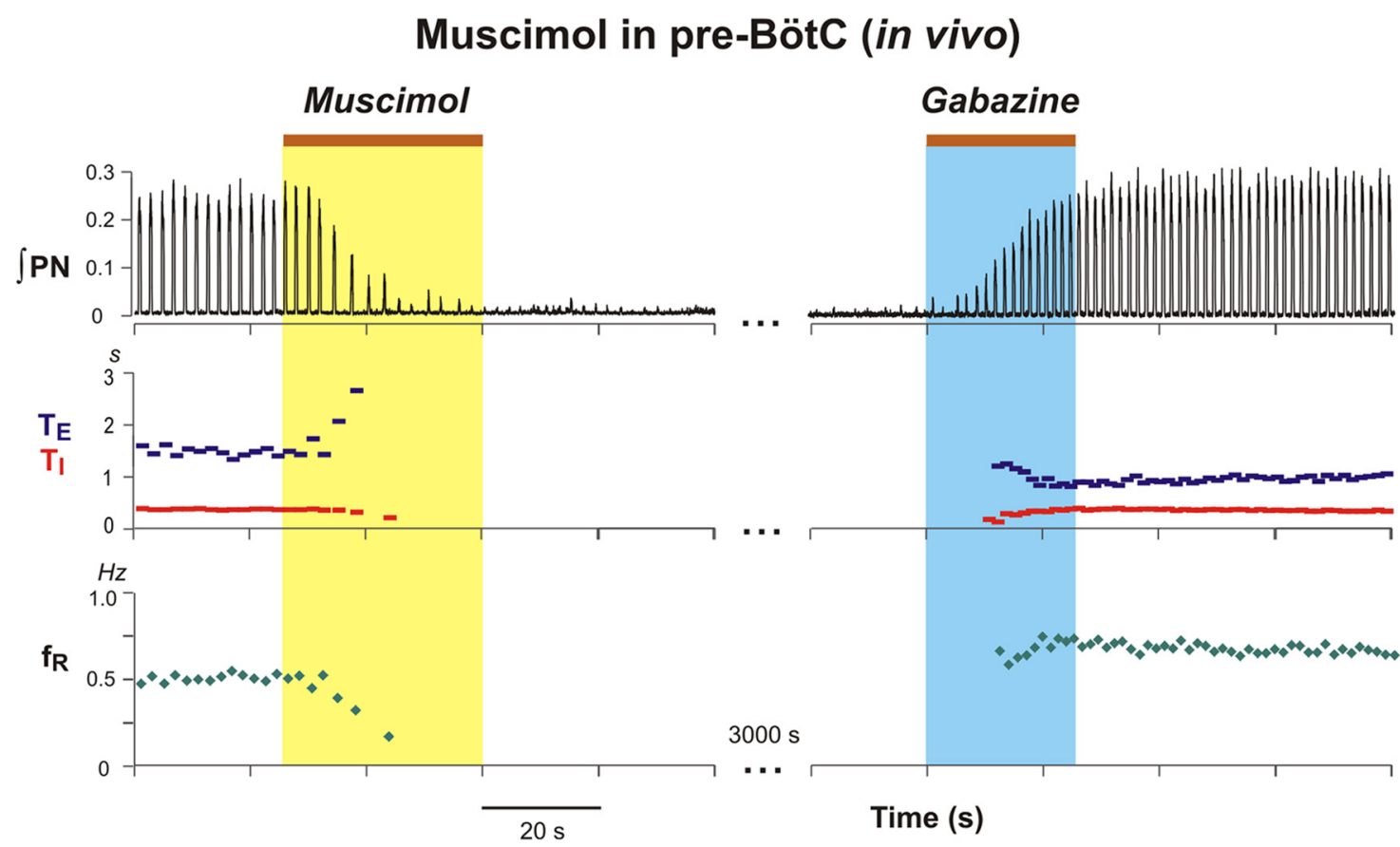

Figure 11. Suppression of rhythmic inspiratory activity by bilateral microinjection of muscimol in the pre-BötC of adult rat in vivo and gabazine antagonism of $\mathrm{GABA}_{\mathrm{A}}$ receptor activation. Muscimol (30 $\mathrm{nl}, 100 \mu \mathrm{M}$ ) microinjection (during yellow shaded area) rapidly reduced integrated PN activity and produced a long-lasting suppression of inspiratory activity that could be restored by bilateral microinjection of gabazine $(110 \mathrm{nl}, 250 \mu \mathrm{M})$ at the same site in the pre-BötC (blue shaded area). $\int \mathrm{PN}$, phrenic nerve integrated activity; $f_{\mathrm{R}}$, respiratory frequency.

vagotomized adult cats (Pierrefiche et al., 1998) demonstrating that bilateral pharmacological disruption of glycinergic synaptic inhibition with strychnine in the preBötC reduces inspiratory discharge amplitude and markedly augments inspiratory discharge frequency or totally abolishes rhythmic phrenic nerve activity. In their studies, simultaneous block of GABA $A_{A}$ ergic and glycinergic inhibition led to sustained tonic discharge on phrenic nerves, indicating disruption of rhythmic inspiratory motor output. Furthermore, results obtained in anesthetized vagotomized adult rabbits by Bongianni et al. (2010) also demonstrated that bilateral block/attenuation of glycinergic inhibition in the pre-BötC augmented inspiratory frequency and reduced inspiratory discharge amplitude. Also, blocking GABAergic inhibition with gabazine in the BötC strongly depressed inspiratory amplitude and frequency leading to apnea as we demonstrated with $\mathrm{GABA}_{\mathrm{A}}$ ergic and glycinergic antagonists in several cases. Moreover, in these studies in rabbits, bilateral microinjection of muscimol in the BötC potently augmented inspiratory discharge frequency and caused loss of rhythmic inspiratory activity that was replaced by tonic discharge of phrenic nerves.

The general conclusions from these previous studies in both vagotomized and vagus-intact animals, similar to our conclusions, are that ongoing neuronal $\mathrm{GABA}_{\mathrm{A}}$ and glycine receptor-mediated postsynaptic inhibition in the pre-BötC and BötC have a major role in controlling the frequency and amplitude of inspiratory circuit activity during eupneic breathing in vivo. Other results obtained by pharmacologically blocking glycinergic inhibition with systemically applied strychnine in perfused rat and mouse brainstem-spinal cord preparations in situ, disturbing synaptic inhibition throughout the respiratory network, also demonstrate major disturbances of respiratory rhythm and disruption of the three-phase respiratory pattern at cellular and circuit levels (Shevtsova et al., 2011, 2014; Richter and Smith, 2014).

All of the above results and conclusions contrast with those from the recent pharmacological study by Janczewski et al. (2013) performed in anesthetized spontaneously breathing, vagus-intact or vagotomized adult rats in vivo. They reported that sequential bilateral microinjections of bicuculline and strychnine in the pre-BötC and then BötC via a ventral surface approach does not significantly disturb inspiratory frequency in vagotomized rats, whereas in vagus-intact rats inspiratory frequency was significantly reduced, accompanied by a prolongation of $T_{1}$ and $T_{E}-$ perturbations that the authors attributed to only suppression of the Breuer-Hering inspiratory inhibitory reflex $(\mathrm{BHIR})$, supported by their results showing that the $\mathrm{BHIR}$ is blocked by antagonizing synaptic inhibition in the pre-BötC. Other results presented suggested that block of inhibition in the pre-BötC and BötC does not disturb generation of laryngeal post-I activity in vagus-intact rats and thus they concluded that inhibitory circuit interactions do not participate in normal three-phase respiratory pattern generation. This result conflicts with our present and previous results (Shevtsova et al., 2011) in situ, where we 


\section{Muscimol in pre-BötC (in situ)}

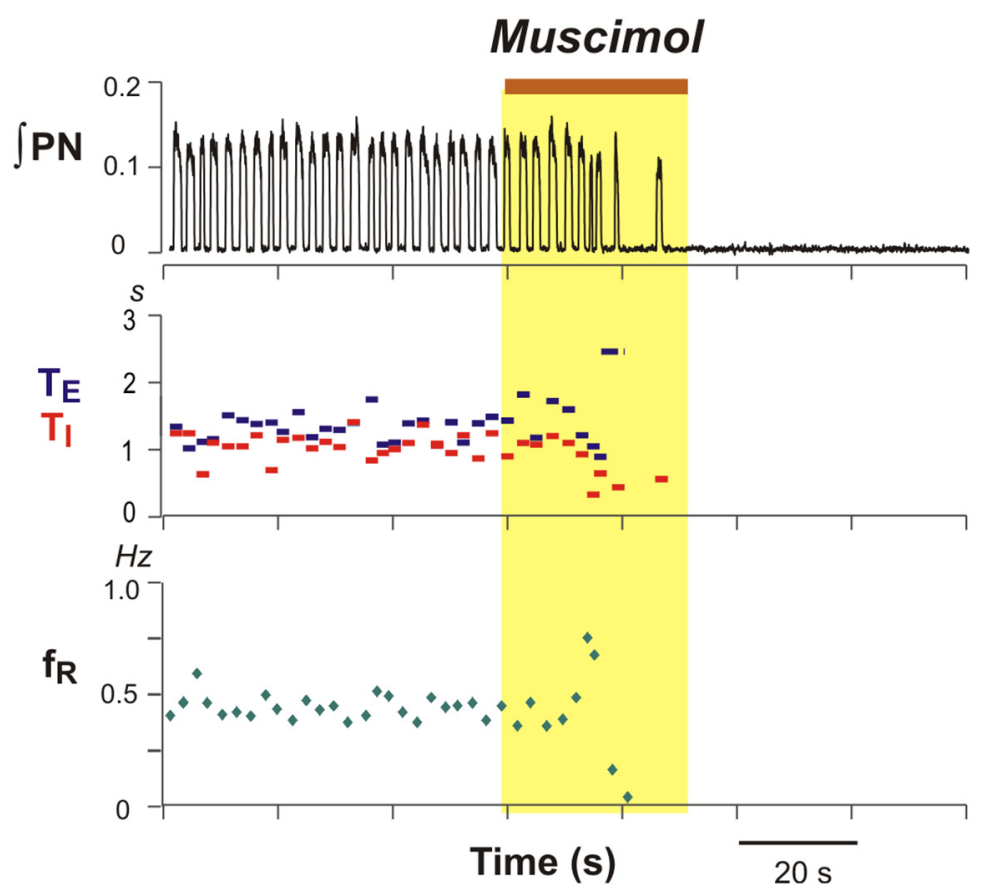

Figure 12. Illustration of prolonged suppression of rhythmic inspiratory activity by bilateral microinjection of muscimol (10 $\mu \mathrm{m})$ in the pre-BötC (yellow shaded area) of juvenile rat perfused brainstem-spinal cord in situ. As in vivo, muscimol rapidly reduces phrenic nerve inspiratory discharge frequency and terminates inspiratory activity. $\int \mathrm{PN}$, phrenic nerve integrated activity; $f_{\mathrm{R}}$, respiratory frequency.

could routinely record post-I activity on cVN or post-I neuronal activity in this unanaesthetized preparation, and found that disruption of synaptic inhibition in the pre-BötC or BötC consistently eliminated post-I activity. Another major result by Janczewski et al. (2013) was that bilateral ablation of the BötC did not significantly perturb inspiratory frequency or inspiratory-expiratory phase durations, from which they concluded that the BötC is not involved at all in generating the three-phase rhythmic respiratory pattern. This clearly contrasts with our results and those of Bongianni et al. (2010) that disrupting inhibition in the BötC can cause apnea, whereas suppressing BötC neuronal activity with muscimol augments inspiratory frequency and can lead to apneustic-like tonic discharge, similar to disrupting inhibition in the pre-BötC. Janczewski et al. (2013) presented data showing a similar augmentation of frequency leading to tonic discharge and disruption of rhythm generation with muscimol injections in the pre-BötC, which is the opposite of ours and previous results that suppressing neuronal activity with muscimol in the pre-BötC potently reduces inspiratory frequency and rapidly terminates inspiratory rhythm generation. Overall Janczewski et al. (2013) concluded that inhibition is not required for a normal eupneic breathing rhythm in vivo except for mediation of the BHIR in vagus-intact animals.

These major discrepancies are not readily resolved. The results obtained with these targeted pharmacological approaches depend critically on accurate site-directed delivery of the inhibitory agonists/antagonists, the ability to antagonize inhibitory postsynaptic receptors on a suffi- cient number of neurons by regional spread of the antagonists/agonists to ultimately perturb motor outputs, and also on regionally confining the pharmacological perturbations. In our experiments, we verified locations of injection sites histologically and we consistently obtained differential, regionally specific perturbations of inspiratory rhythm: slowing of the rhythm in all cases by inhibitory antagonists in BötC, and contrasting augmented inspiratory frequency with antagonists in pre-BötC. Our reconstructed microinjection sites appear to be identical to the sites reconstructed by Janczewski et al. (2013, their Fig. 1; compare to our Fig. 1). In our in vivo experiments using a similar ventral surface approach in adult anesthetized rats, we used identical concentrations and ejection volumes of antagonists, which we also showed, at least for gabazine, had pharmacologically effective $\mathrm{GABA}_{A}$ receptor antagonist-agonist interactions (Fig. 11). With our targeting procedures, which included mapping of characteristic neuronal activity profiles, as well as differential respiratory and blood pressure responses to local L-Glu microinjections, we also consistently obtained regionally specific perturbations with muscimol microinjections at targeted sites: reduced inspiratory frequency and apnea in the pre-BötC, but augmented inspiratory frequency in the BötC. This specificity also suggests that it was possible to confine actions of the agonists/antagonists to the targeted region. Differences in the numbers of neurons and inhibitory synapses affected by local diffusion of the antagonists might contribute, although assuming accuracy of targeting, we would expect similar 


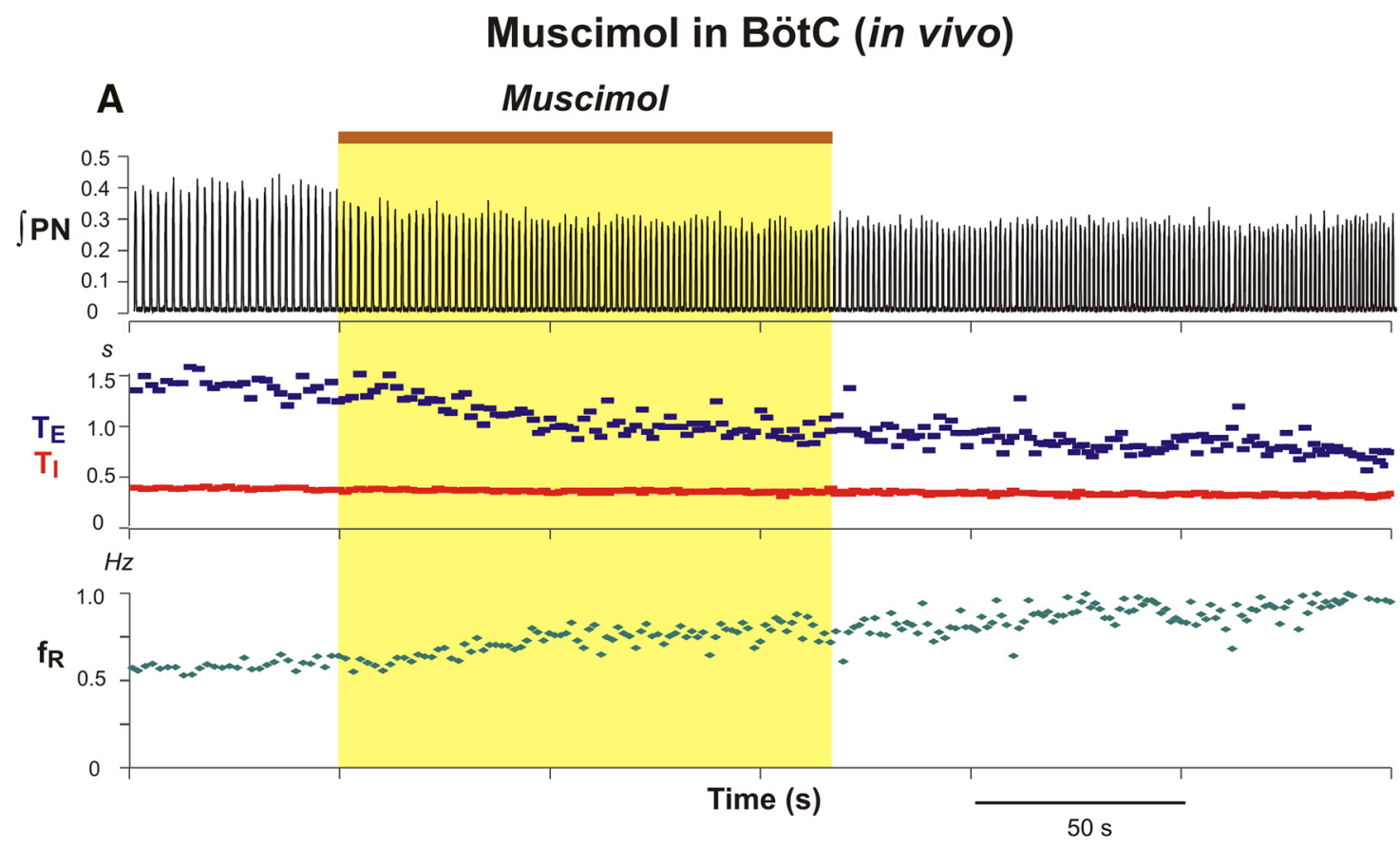

Normalized values $(n=7)$

B
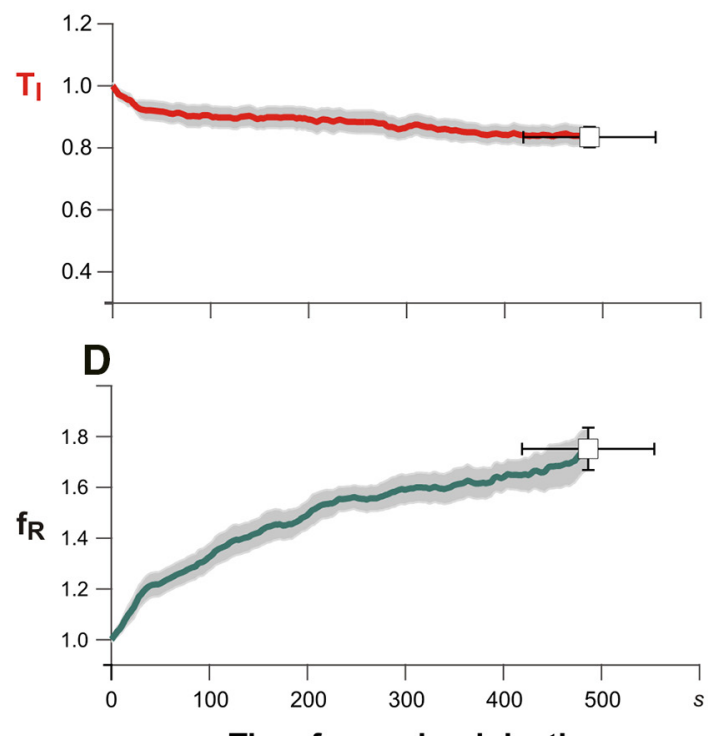

C
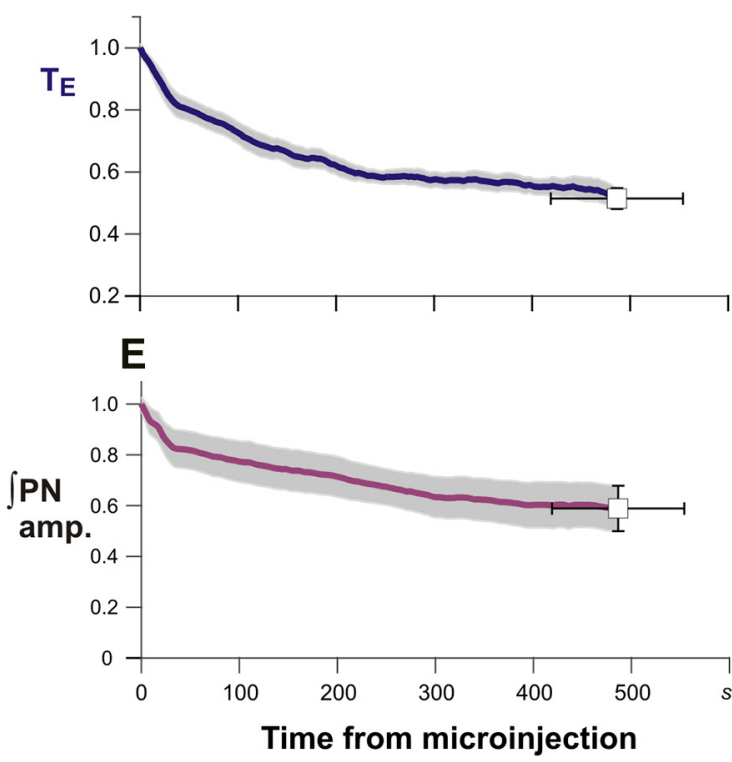

Figure 13. Perturbation of inspiratory activity by bilateral microinjection of muscimol in the BötC of anesthetized adult rat in vivo. $\boldsymbol{A}$, Muscimol (30 $\mathrm{nl}, 100 \mu \mathrm{M}$ ) slowly microinjected (brown bar at the top and yellow rectangle) in the BötC augments PN frequency due to a reduction of $\mathrm{T}_{\mathrm{E}}$. $\boldsymbol{B}$ - $\boldsymbol{E}$, Mean time series for group data $(n=7)$ summarizing perturbations of respiratory rhythm and motor output pattern parameters. Solid colored curves are mean time and normalized parameter values and gray bands are $\pm 1 \mathrm{SEM}$ of $T_{1}(\boldsymbol{B}), \mathrm{T}_{E}(\boldsymbol{C})$, respiratory frequency $\left(\mathrm{f}_{\mathrm{R}} ; \boldsymbol{D}\right)$, and integrated inspiratory activity amplitude $(\boldsymbol{E})$ computed from recordings of integrated phrenic nerve activity as in previous figures.

perturbations because ejection volumes and antagonist concentrations used in vivo were identical.

\section{Relation to studies using selective targeting of inhibitory neurons}

In a recent study using optogenetic approaches to selectively stimulate or inhibit pre-BötC glycinergic neurons by virally-transduced expression of photosensitive opsins channelrhodopsin (ChR2) or archaerhodopsin in spontaneously breathing adult mice in vivo, Sherman et al. (2015) found that optical stimulation of this subset of inhibitory neurons in pre-BötC in vivo can terminate inspiration, delay the onset of inspiration with photoactivation during the expiratory phase, and produce long-lasting apnea 


\section{Muscimol in BötC (in situ)}
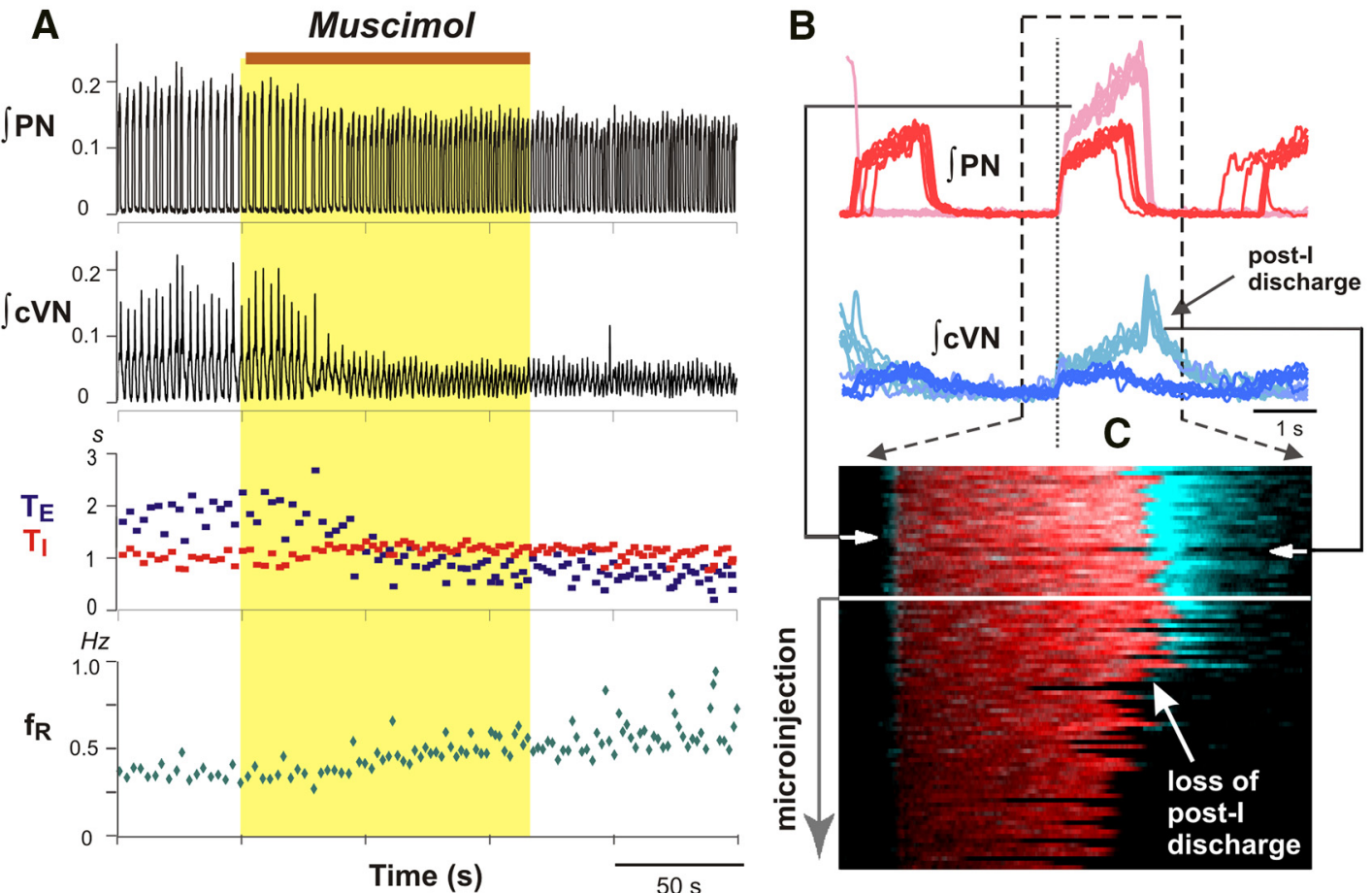

Normalized values $(n=5)$

D
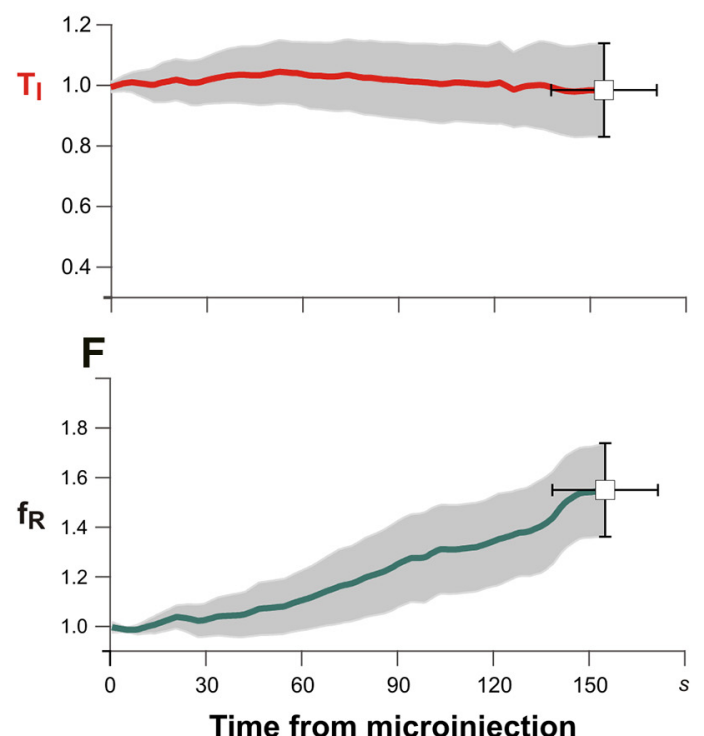

E

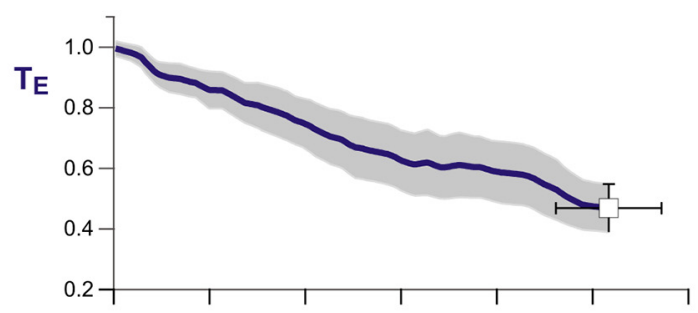

G

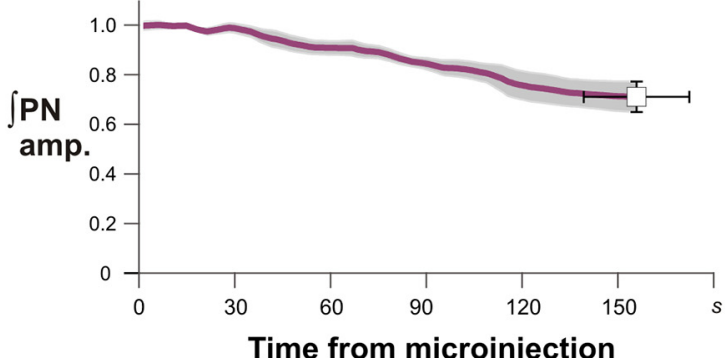

Figure 14. Disturbances of respiratory pattern including elimination of post-inspiratory activity by bilateral microinjection of muscimol $(10 \mu \mathrm{M})$ in the BötC of juvenile rat perfused brainstem-spinal cord in situ. $\boldsymbol{A}$, Representative example of augmented $\int \mathrm{PN}$ inspiratory discharge frequency and pronounced reduction of cVN integrated $\left(\int \mathrm{cVN}\right)$ activity amplitude due to suppression of post-I activity. Overlaid traces in $\boldsymbol{B}$ and time-series raster plot in $\mathbf{C}$ of consecutive respiratory cycles aligned to inspiratory onset (indicated by vertical dotted line in $\boldsymbol{B}$ ) illustrate perturbations of cVN post-I discharge during BötC muscimol microinjection in another experiment. $\boldsymbol{B}$, Integrated PN (red traces) and cVN (blue traces) activity are shown before (lighter red and lighter blue traces, respectively) and after (dark red and dark blue traces) muscimol microinjection. Aligned traces of $\int P N$ (red) and $\int c V N$ (blue) are shown in raster plot in $\boldsymbol{C}$ with the loss of post-I cVN discharge indicated (white arrow), which is also clearly seen from the $\int \mathrm{cVN}$ traces (dark blue) above. $\boldsymbol{D}-\mathbf{G}$, Mean time series for group data $(n=6)$ summarizing perturbations of respiratory rhythm/pattern parameters. Solid colored curves are mean time and parameter values and gray bands are \pm 1 SEM of normalized $T_{1}(\boldsymbol{D}), \mathrm{T}_{E}(\boldsymbol{E})$, inspiratory frequency $\left(\mathrm{f}_{\mathrm{R}} ; \boldsymbol{F}\right)$, and integrated inspiratory activity amplitude $(\boldsymbol{G})$ computed from recordings of integrated PN activity. 
with prolonged photostimulation. Conversely, prolonged photoinhibition augmented the amplitude and frequency of inspiratory activity and could reverse reflex-induced apneas. This approach can provide important information on dynamic perturbations resulting from augmentation or loss of inhibitory neuron function (Abdala et al., 2015) and their results demonstrate that at least pre-BötC glycinergic neuron activity can strongly modulate ongoing inspiratory rhythm and pattern generation. The authors concluded, however, that glycinergic inhibition is not essentially involved in rhythmogenesis despite these perturbations because inspiratory rhythm persisted after photoinhibition, although as the authors discuss, it is unclear whether sufficient numbers of glycinergic neurons were optically silenced to reveal full affects of pre-BötC glycinergic neuron activity on rhythm generation.

Another important consideration for interpreting the significance of these results is that silencing pre-BötC glycinergic neurons may not be analogous to pharmacologically blocking even postsynaptic glycinergic receptors, particularly because relevant glycinergic synapses may originate from neurons outside of the pre-BötC such as BötC neurons not transduced in sufficient numbers with viral vectors targeted to the pre-BötC. These optogenetic results also cannot be readily compared with the effects of pharmacological blockade of both glycinergic and $\mathrm{GABA}_{A}$ ergic postsynaptic inhibition. As known, preBötC contains inhibitory neurons with each type of transmission, as well as neurons coexpressing both GABA and glycine (Schreihofer et al., 1999; Koizumi et al., 2013), so all these neuron types would have to be optically inhibited to fully evaluate the functional role of local inhibitory circuits in the pre-BötC. The augmented inspiratory frequency that we observed with disrupting inhibition in the pre-BötC, which has also been observed by pharmacologically attenuating only glycinergic inhibition with strychnine (Pierrefiche et al., 1998) as discussed above, is a common result of both pharmacological and optical approaches. However, inspiratory amplitude was always markedly reduced, not augmented, by pharmacologically perturbing postsynaptic inhibition in the pre-BötC, suggesting that more complex inhibitory interactions in pre-BötC inspiratory rhythm generation circuits are revealed by the pharmacological approaches. Experiments were not presented by Sherman et al. (2015) on photostimulation/inhibition of glycinergic neurons in the BötC. Optical stimulation of BötC neurons transduced with ChR2, although not selectively in GABAergic and glycinergic neurons but likely including these neurons in these studies, strongly suppresses inspiratory activity in contrast to the powerful augmentation of frequency with photostimulation of pre-BötC neurons in anesthetized rats in vivo (Alsahafi et al., 2015). These observations are analogous to our results with local glutamate microinjections, which also do not selectively stimulate specific neuronal phenotypes in these regions.

\section{Normal eupneic rhythm generation in the absence of synaptic inhibition in vivo?}

A continuing debate is whether a normal eupneic inspiratory rhythm and respiratory pattern can be generated without synaptic inhibition in the pre-BötC in the intact system where the pre-BötC interacts with the BötC and other sources of phasic/tonic synaptic inhibition. We show that disrupting pre-BötC synaptic inhibition leads either to higher frequency oscillations accompanied by a large reduction in the amplitude of pre-BötC/phrenic inspiratory activity or to tonic phrenic nerve activity in some causes, indicating that a normal eupneic inspiratory rhythm generally does not occur in the absence of synaptic inhibition. The variable persistence of inspiratory rhythmic activity in other cases, albeit with large disturbances of the inspiratory rhythm and inspiratory-expiratory pattern, indicates that either we did not sufficiently block postsynaptic inhibition in the pre-BötC with our targeted pharmacological approach in these cases, or some form of inspiratory rhythm generation occurs after disruption of $\mathrm{GABA}_{A}$ ergic and glycinergic inhibition in the intact system. The former is a technical problem that is difficult to solve. This would require extensive local intracellular recordings to analyze endogenous postsynaptic inhibitory potentials simultaneously with the pharmacological perturbations. In our approach, we limited the microinjection volumes of the pipette solution containing inhibitory antagonists, in an attempt to achieve sitespecificity of the perturbations. This may have precluded sufficient inhibitory receptor block throughout the local circuits to cause complete disruption of inspiratory rhythm generation in these cases. On the other hand, pre-BötC excitatory neurons and circuits have intrinsic rhythmogenic properties (Smith et al., 2007; St. John et al., 2009; Richter and Smith, 2014), so it is possible that some form of inspiratory rhythm generation, albeit abnormal, can persist in the absence of synaptic inhibition in the intact juvenile/adult system, as occurs in the neonatal $\mathrm{rat} / \mathrm{mouse}$ pre-BötC isolated in slices in vitro.

Our models of excitatory and inhibitory pre-BötC circuits and their interactions with BötC inhibitory neurons in the intact system incorporate intrinsic rhythmogenic properties in the excitatory pre-BötC population (Rybak et al., 2004, 2007; Smith et al., 2007; Rubin et al., 2009). These models indicate that depending on the initial excitation state of the excitatory kernel neurons, the endpoint after complete block of postsynaptic inhibition can be either tonic activity of pre-BötC neurons at high levels of excitation or rhythmic activity at lower excitation levels. It is clear that to answer the question of whether any form of inspiratory rhythm generation can occur, intracellular recordings will have to be used to monitor the level of membrane potential and activity patterns during and after local block of postsynaptic inhibition.

\section{Disorganization of the three-phase respiratory pattern after disrupting postsynaptic inhibition}

The three-phase organization of the respiratory pattern at least in situ was disrupted accompanying the large disturbances of inspiratory rhythm by blocking inhibition in 
the pre-BötC or BötC. This confirms that the normal eupneic breathing pattern with appropriately coordinated spinal and cranial motoneuron activity relies on synaptic inhibition in BötC and pre-BötC circuits. These inhibitory circuits normally operate with pontine and other excitatory inputs (Rybak et al., 2004; Smith et al., 2007; Dutschmann and Dick, 2012). The pontine Kölliker-Fuse nucleus projects heavily to both the pre-BötC and BötC affecting their inhibitory interactions. Specifically, pontine input is required to generate post-I activity (Rybak et al., 2004; Smith et al., 2007; Dutschmann and Dick, 2012) so the observed loss of this activity in part reflects disruption of the balance of excitatory and inhibitory interactions. Recent experiments in a transected perfused rat brainstem preparation have confirmed the critical role of the pons in three-phase respiratory pattern generation (Jones and Dutschmann, 2016). Previously it has been demonstrated that blocking glycinergic inhibition systemically causes a shift of post-I neuron activity into the inspiratory phase, due to phasic excitation of post-I neurons that is normally shunted by inspiratory phase inhibition (Richter and Smith, 2014) and this shift causes abnormal glottal constriction during inspiration (Dutschmann and Paton, 2002). This also implies that the shifted post-I inhibitory neurons would provide abnormally timed inhibition during the inspiratory phase, possibly including to inspiratory neurons in bulbospinal circuits downstream from the BötC and pre-BötC, which could contribute to the large reductions of inspiratory amplitude and attenuation of PN ramping inspiratory discharge observed after disrupting inhibition. The loss of the three-phase pattern is predicted from models (Rybak et al., 2004, 2007; Smith et al., 2007; Shevtsova et al., 2011, 2014) after inhibitory block in the BötC, where post-I inhibitory neuronal activity is presumed generated. Our results that the three-phase pattern is also disrupted after blocking inhibition in the pre-BötC may reflect the known presence of post-I neurons in the pre-BötC (Schwarzacher et al., 1995; Alheid and McCrimmon, 2008), and shows that the pre-BötC also plays a role in three-phase pattern generation, conceivably by either interactions with the BötC and/or by local inhibitory circuit interactions, which need to be delineated.

\section{Role of BötC circuits and synaptic inhibition in respiratory pattern generation}

A fundamental role for BötC neurons in respiratory pattern generation has been questioned by Janczewski et al. (2013) as noted above. Their conclusion that this region plays no role is incompatible with the present and previous results showing that augmenting BötC neuron activity (by glutamate microinjections) suppresses pre-BötC inspiratory activity, whereas suppressing BötC activity (by muscimol) augments pre-BötC inspiratory rhythm. The BötC is known to contain major populations of active excitatory and inhibitory expiratory post-I and aug-E neurons, and this region is critical for generating post-I activity (Burke et al., 2010). Excitatory BötC neurons are thought to be a major source of expiratory activity in the respiratory network including for generation of post-I pre- motoneuron activity. BötC glycinergic and GABAergic expiratory interneurons are proposed to provide widely distributed network synaptic inhibition during expiration (Jiang and Lipski, 1990; Tian et al., 1999a,b; Ezure et al., 2003a,b). These postulated inhibitory connections include to pre-BötC excitatory and inhibitory inspiratory neurons to provide phasic synaptic inhibition orchestrating rhythmic alternation between expiratory and inspiratory activity in the network. Mutual inhibitory synaptic interactions between BötC inhibitory (post-I and aug-E) expiratory neurons and inhibitory inspiratory (e.g., early-l) pre-BötC neurons have been proposed to coordinate generation of the three-phase pattern of neuronal activity during eupneic breathing (Rybak et al., 2004, 2007; Smith et al., 2007; Rubin et al., 2009; Shevtsova et al., 2011, 2014; Richter and Smith, 2014). Based on these proposed interactions, augmenting BötC activity or disrupting postsynaptic inhibition within the BötC should suppress rhythmic activity in the pre-BötC, assuming in each case that BötC neurons shift from phasic to tonic activity. Correspondingly, suppressing BötC activity should augment inspiratory discharge frequency, and depending on the level of ongoing pre-BötC excitation, this can lead to tonic inspiratory activity. Both of the above are features of the present experimental results.

We note that the projections and postsynaptic targets of BötC and pre-BötC inhibitory interneurons, particularly mutual inhibitory connections between these neurons, have not been mapped structurally. Establishing these connections remains an important problem. Nevertheless, our results are consistent with mutual interactions and a fundamental role of the BötC and its expiratory inhibitory neurons in three-phase respiratory pattern generation and control of inspiratory rhythm.

\section{References}

Abdala AP, Paton JFR, Smith JC (2015) Defining inhibitory neurone function in respiratory circuits: opportunities with optogenetics? J Physiol 593:3033-3046. CrossRef Medline

Alheid GF, McCrimmon DR (2008) The chemical neuroanatomy of breathing. Respir Physiol Neurobiol 164:3-11. CrossRef Medline

Alsahafi Z, Dickson CT, Pagliardini S (2015) Optogenetic excitation of preBötzinger complex neurons potently drives inspiratory activity in vivo. J Physiol 593:3673-3692. CrossRef Medline

Baekey DM, Molkov YI, Paton JFR, Rybak IA, Dick TE (2010) Effect of baroreceptor stimulation on the respiratory pattern: insights into respiratory-sympathetic interactions. Respir Physiol Neurobiol 174:135-145. CrossRef

Bongianni F, Mutolo D, Cinelli E, Pantaleo T (2010) Respiratory responses induced by blockades of GABA and glycine receptors within the Bötzinger complex and the pre-Bötzinger complex of the rabbit. Brain Res 1344:134-147. CrossRef Medline

Burke PGR, Abbott SBG, McMullan S, Goodchild AK, Pilowsky PM (2010) Somatostatin selectively ablates post-inspiratory activity after injection into the Bötzinger complex. Neuroscience 167:528539. CrossRef

Dutschmann M, Dick TE (2012) Pontine mechanisms of respiratory control Compr Physiol 2:2443-2469. CrossRef Medline

Dutschmann, M, Paton, JF (2002) Glycinergic inhibition is essential for co-ordinating cranial and spinal respiratory motor outputs in the neonatal rat. J Physiol 543:643-653. CrossRef

Ezure K, Tanaka I, Kondo M (2003a) Glycine is used as a transmitter by decrementing expiratory neurons of the ventrolateral medulla in the rat. J Neurosci 23:8941-8948. Medline 
Ezure K, Tanaka I, Saito Y (2003b) Brainstem and spinal projections of augmenting expiratory neurons in the rat. Neurosci Res 45:4151. Medline

Feldman JL, Del Negro CA, Gray PA (2013) Understanding the rhythm of breathing: so near, yet so far. Ann Rev Physiol 75:423452. CrossRef Medline

Grillner S (2006) Biological pattern generation: the cellular and computational logic of networks in motion. Neuron 52:751-766. CrossRef Medline

Grundy EM, Chakrabarti MK, Whitwam JG (1986) Efferent phrenic nerve activity during induced changes in arterial pressure. $\mathrm{Br} J$ Anaesth 58:1414-1421. Medline

Hopp FA, Seagard JL (1998) Respiratory responses to selective blockade of carotid sinus baroreceptors in the dog. Am J Physiol 275:R10-R18. Medline

Janczewski WA, Tashima A, Hsu P, Cui Y, Feldman, JL (2013) Role of inhibition in respiratory pattern generation. J Neurosci 33:54545465. CrossRef Medline

Jiang C, Lipski J (1990) Extensive monosynaptic inhibition of ventral respiratory group neurons by augmenting neurons in the Bötzinger complex in the cat. Exp Brain Res 81:639-648. Medline

Johnson SM, Koshiya N, Smith JC (2001) Isolation of the kernel for respiratory rhythm generation in a novel preparation: the preBötzinger complex “island." J Neurophysiol 85:1772-1776. Medline

Jones SE, Dutschmann M (2016) Testing the hypothesis of neurodegeneracy in respiratory network function with a priori transected arterially perfused brainstem preparation of rat. J Neurophysiol. Advance online publication. Feb. 17 [10.1152/jn.01073.2015]

Kanjhan R, Lipski J, Kruszewska B, Rong WA (1995) Comparative study of pre-sympathetic and Bötzinger neurons in the rostral ventrolateral medulla (RVLM) of the rat. Brain Res 699:19-32. Medline

Koizumi H, Koshiya N, Chia JX, Cao F, Nugent J, Zhang R, Smith JC (2013) Structural-functional properties of identified excitatory and inhibitory interneurons within pre-Bötzinger complex respiratory microcircuits. J Neurosci 33:2994-3009. CrossRef Medline

Koshiya N, Smith JC (1999) Neuronal pacemaker for breathing visualized in vitro. Nature 400:360-363. CrossRef Medline

Kuwana SI, Tsunekawa N, Yanagawa Y, Okada Y, Kuribayashi J, Obata K (2006) Electrophysiological and morphological characteristics of GABAergic respiratory neurons in the mouse preBötzinger complex. Eur J Neurosci 23:667-674. CrossRef

Lindsey BG, Rybak IA, Smith JC (2012) Computational models and emergent properties of respiratory neural networks. Compr Physiol 2:1619-1670. CrossRef Medline

Lipski J, Kanjhan R, Kruszewska B, Rong WA, Smith M (1996) Pre-sympathetic neurones in the rostral ventrolateral medulla of the rat: electrophysiology, morphology and relationship to adjacent neuronal groups. Acta Neurobiol Experiment 56:373-384.

Molkov YI, Borgmann A, Zhang R, Rybak IA, Smith JC (2012) Analysis of excitatory and inhibitory interactions at high temporal resolution in core circuits of the respiratory CPG. BMC Neurosci 13:39. CrossRef

Moraes DJ, Zoccal DB, Machado BH (2012) Sympathoexcitation during chemoreflex active expiration is mediated by l-glutamate in the RVLM/Bötzinger complex of rats. J Neurophysiol 108:610-623. CrossRef Medline

Morgado-Valle C, Baca SM, Feldman JL (2010) Glycinergic pacemaker neurons in preBötzinger complex of neonatal mouse. J Neurosci 30:3634-3639. CrossRef Medline

Paton JFR (1996) A working heart-brainstem preparation of the mouse. J Neurosci Methods 65:63-68. Medline

Paton JF, Abdala AP, Koizumi H, Smith JC St-John WM (2006) Respiratory rhythm generation during gasping depends on persistent sodium current. Nat Neurosci 9:311-313. CrossRef Medline

Pierrefiche O, Schwarzacher SW, Bischoff, AM, Richter DW (1998) Blockade of synaptic inhibition within the pre-Bötzinger complex in the cat suppresses respiratory rhythm generation in vivo. J Physiol 509:245-254. CrossRef
Richter DW (1996) Neural regulation of respiration: rhythmogenesis and afferent control. In: Comprehensive human physiology: from cellular mechanism to integration, (Gregore R, Windhorts U, eds), pp. 2079-2095. Berlin: Springer Verlag.

Richter DW, Seller H (1975) Baroreceptor effects on medullary respiratory neurones of the cat. Brain Res 86:168-171. Medline

Richter DW, Smith JC (2014) Respiratory rhythm generation in vivo. Physiology 29:58-71. CrossRef Medline

Rubin JE, Shevtsova NA, Ermentrout GB, Smith JC, Rybak IA (2009) Multiple rhythmic states in a model of the respiratory central pattern generator. J Neurophysiol 101:2146-2165. CrossRef Medline

Rybak IA, Abdala AP, Markin SN, Paton JFR, Smith JC (2007) Spatial organization and state-dependent mechanisms for respiratory rhythm and pattern generation. Prog Brain Res 165:201-220. CrossRef Medline

Rybak IA, Shevtsova NA, Paton JF, Dick TE, St-John WM, Mörschel M., Dutschmann M (2004) Modeling the ponto-medullary respiratory network. Respir Physiol Neurobiol 143:307-319. CrossRef Medline

Schmid K, Foutz AS, Denavit-Saubié M (1996) Inhibitions mediated by glycine and GABAA receptors shape the discharge pattern of bulbar respiratory neurons Brain Res 710:150-160. Medline

Schreihofer AM, Stornetta RL, Guyenet PG (1999) Evidence for glycinergic respiratory neurons: Bötzinger neurons express mRNA for glycinergic transporter 2. J Comp Neurol 407:583-597. Medline

Schwarzacher SW, Smith JC, Richter DW (1995) Pre-Bötzinger complex in the cat. J Neurophysiol 73:1452-1461. Medline

Sherman D, Worrell, Cui Y, Feldman JL (2015) Optogenetic perturbation of preBötzinger complex inhibitory neurons modulates respiratory pattern. Nat Neurosci 18:408-414 CrossRef Medline

Shevtsova NA, Manzke T, Molkov YI, Bischoff A, Smith JC, Rybak IA, Richter DW (2011) Computational modelling of 5-HT receptormediated reorganization of the brainstem respiratory network. Eur J Neurosci 34:1276-1291. CrossRef Medline

Shevtsova NA, Büsselberg D, Molkov YI, Bischoff AM, Smith JC, Richter DW, Rybak IA (2014) Effects of glycinergic inhibition failure on respiratory rhythm and pattern generation. Prog Brain Res 209:25-38. CrossRef Medline

Smith JC, Abdala AP, Borgmann A, Rybak IA, Paton JF (2013) Brainstem respiratory networks: building blocks and microcircuits. Trends Neurosci 36:152-162. CrossRef Medline

Smith JC, Abdala AP, Koizumi H, Rybak IA, Paton JF (2007) Spatial and functional architecture of the mammalian brain stem respiratory network: a hierarchy of three oscillatory mechanisms. J Neurophysiol 98:3370-3387. CrossRef Medline

Smith JC, Abdala AP, Rybak IA, Paton JF (2009) Structural and functional architecture of respiratory networks in the mammalian brainstem. Philos Trans R Soc Lond B Biol Sci 364:2577-2587. CrossRef Medline

Smith JC, Ellenberger HH, Ballanyi K, Richter DW, Feldman JL (1991) Pre-Bötzinger complex: a brainstem region that may generate respiratory rhythm in mammals. Science 254:726-729. Medline

St. John WM, Stornetta RL, Guyenet PG, Paton JF (2009) Location and properties of respiratory neurones with putative intrinsic bursting properties in the rat in situ. J Physiol 587:3175-3188. CrossRef Medline

Tian, GF, Peever JH, Duffin J (1999a) Bötzinger complex, bulbospinal expiratory neurons monsynaptically inhibit ventral respiratory group neurons in the decerebrate rat. Exp Brain Res 124: 173-180

Tian GF, Peever JH, Duffin J (1999b) Mutual inhibition between Bötzinger-complex bulbospinal expiratory neurons detected with cross-correlation in the decerebrate rat. Exp Brain Res 125:440-446.

Virkki A, Polod O, Gyllenberg M, Aittokallio T (2007) Can carotid body perfusion act as a respiratory controller? J Theor Biol 249:737-748. CrossRef Medline

Winter S, Fresemann J, Schnell C, Oku Y, Hirrlinger J, Hülsmann S (2009) Glycinergic interneurons are functionally integrated into the inspiratory network of mouse medullary slices. Pflugers Arch 458: 59-469. CrossRef 